\title{
EXISTENCE OF VERY WEAK SOLUTIONS OF DOUBLY NONLINEAR PARABOLIC EQUATIONS WITH MEASURE DATA
}

\author{
Stefan Sturm \\ Paris-Lodron-Universität Salzburg, Fachbereich Mathematik \\ 5020 Salzburg, Austria; Stefan.Sturm@sbg.ac.at
}

\begin{abstract}
We deal with a Cauchy-Dirichlet problem with homogeneous boundary conditions on the parabolic boundary of a space-time cylinder for doubly nonlinear parabolic equations, whose prototype is

$$
\partial_{t} u-\operatorname{div}\left(|u|^{m-1}|D u|^{p-2} D u\right)=\mu
$$

with a non-negative Radon measure $\mu$ on the right-hand side. Here, the doubly degenerate $(p \geq 2$, $m \geq 1)$ and singular-degenerate $\left(p \in\left(\frac{2 n}{n+2}, 2\right), m \geq 1\right)$ cases are considered. The central objective is to establish the existence of a solution in the sense of distributions (see Theorem 1.4). The constructed solution is obtained by a limit of approximations, i.e. we use solutions of regularized Cauchy-Dirichlet problems and pass to the limit to receive a solution for the original CauchyDirichlet problem.
\end{abstract}

\section{Introduction and main result}

We study the existence of solutions of the Cauchy-Dirichlet problem for doubly nonlinear parabolic equations with measure data. Such equations arise in the field of plasma physics, ground water problems, or the motion of viscous fluids, but also in the modeling of an ideal gas flowing isoentropically in a homogeneous porous medium.

1.1. Setting. In this paper, we consider nonhomogeneous Cauchy-Dirichlet problems for a class of doubly nonlinear parabolic equations

$$
\partial_{t} u-\operatorname{div}(\mathbf{A}(x, t, u, D u))=\mu
$$

in a space-time cylinder $E_{T}:=E \times(0, T)$, where $E \subset \mathbf{R}^{n}$ is a bounded, open domain, $n \geq 2, T>0$, and $\mu \in \mathcal{M}^{+}\left(E_{T}\right)$ is a non-negative Radon measure on $E_{T}$ with finite total mass $\mu\left(E_{T}\right)<\infty$. The aim of this article is to establish the existence of a solution in the sense of distributions for Cauchy-Dirichlet problems of the form

$$
\begin{cases}\partial_{t} u-\operatorname{div}(\mathbf{A}(x, t, u, D u))=\mu & \text { in } E_{T}, \\ u=0 & \text { on } \Gamma_{T},\end{cases}
$$

where $\Gamma_{T}:=[\bar{E} \times\{0\}] \cup[\partial E \times(0, T)]$ denotes the parabolic boundary of $E_{T}$. Throughout this paper, the vector field $\mathbf{A}: E_{T} \times \mathbf{R} \times \mathbf{R}^{n} \rightarrow \mathbf{R}^{n}$ is assumed to be measurable with respect to $(x, t) \in E_{T}$ for all $(u, \xi) \in \mathbf{R} \times \mathbf{R}^{n}$ and continuous with respect to $(u, \xi) \in \mathbf{R} \times \mathbf{R}^{n}$ for almost every $(x, t) \in E_{T}$. Moreover, we want $\mathbf{A}$ to satisfy the ellipticity condition

$$
\mathbf{A}(x, t, u, \xi) \cdot \xi \geq C_{0}|u|^{m-1}|\xi|^{p}
$$

https://doi.org/10.5186/aasfm.2017.4255

2010 Mathematics Subject Classification: Primary 35K55, 35K20.

Key words: Doubly nonlinear parabolic equations, existence, measure data. 
as well as the growth condition

$$
|\mathbf{A}(x, t, u, \xi)| \leq C_{1}|u|^{m-1}|\xi|^{p-1}
$$

for any $u \in \mathbf{R}, \xi \in \mathbf{R}^{n}$, and almost every $(x, t) \in E_{T}$, where $C_{0}>0$ and $C_{1}>0$ are fixed constants, $p>\frac{2 n}{n+2}$ and $m \geq 1$. Note that the lower bound on $p$ is a typical requirement in the regularity theory for nonlinear parabolic equations (see $[9$, Chap. V, Sec. $3 \& 5]$ ). For $p=2$ or $m=1$, one can retrieve the well-known structure conditions for porous medium type and $p$-Laplacian equations, see e.g. [10, p. 6] and [10, p. 5], respectively. In this regard, (1.3) and (1.4) are their natural extension. Further, we need to restrict ourselves to the case

$$
1<p-\frac{n}{n(\alpha+1)+1}
$$

where $\alpha$ will be defined in (1.8). This constraint is customary, too, and its relevance will be dicsussed in Remark 4.3. Finally, we expect the monotonicity assumption

$$
\begin{aligned}
& {\left[\mathbf{A}\left(x, t, u, \xi_{1}\right)-\mathbf{A}\left(x, t, u, \xi_{2}\right)\right] \cdot\left(\xi_{1}-\xi_{2}\right)} \\
& \geq C_{2}|u|^{m-1}\left|\xi_{1}-\xi_{2}\right|^{\lambda}\left[\left|\xi_{1}-\mathbf{b}(x, t, u)\right|^{p}+\left|\xi_{2}-\mathbf{b}(x, t, u)\right|^{p}\right]^{\frac{p-\lambda}{p}}
\end{aligned}
$$

to hold for any $u \in \mathbf{R}, \xi_{1}, \xi_{2} \in \mathbf{R}^{n}$ with $\xi_{1} \neq \xi_{2}$, and almost every $(x, t) \in E_{T}$ with a fixed constant $C_{2}>0$ and a function $\mathbf{b}: E_{T} \times \mathbf{R} \rightarrow \mathbf{R}^{n}$ which is required to be measurable with respect to $(x, t) \in E_{T}$ for all $u \in \mathbf{R}$ and continuous with respect to $u \in \mathbf{R}$ for almost every $(x, t) \in E_{T}$. Here, $\lambda:=2$ in the case $p \in\left(\frac{2 n}{n+2}, 2\right)$, and $\lambda:=p$ in the case $p \geq 2$. Note that this is the common monotonicity condition (see [16, p. 787]).

1.2. Doubly nonlinear parabolic equations. The model example for equations treated in the sequel is given by the doubly nonlinear parabolic equation

$$
\partial_{t} u-\operatorname{div}\left(|u|^{m-1}|D u|^{p-2} D u\right)=\mu \quad \text { in } E_{T},
$$

whose modulus of ellipticity is $|u|^{m-1}|D u|^{p-2}$. For $p>2, m>1$, this quantity vanishes if $u$ or $|D u|$ become 0 , which is why we call the equation doubly degenerate, whereas in the case that $p \in\left(\frac{2 n}{n+2}, 2\right), m>1$, the coefficient $|D u|^{p-2}$ tends to $\infty$ and $|u|^{m-1} \rightarrow 0$ as $|u| \rightarrow 0,|D u| \rightarrow 0$ such that the equation is singular-degenerate. This classification can also be found in [13, p. 23]. The degenerate-singular $(p>2$, $0<m<1)$ and doubly singular case $\left(p \in\left(\frac{2 n}{n+2}, 2\right), 0<m<1\right)$ will be postponed to a forthcoming article.

Lately, several authors examined doubly nonlinear parabolic equations both because of their physical and mathematical interest, though, substantial parts of the recent research were not on equations of the above general form, but rather on specific examples like (1.7) with either $\mu \equiv 0, p+m=3$, or other simplifications of (1.1). For instance, Hölder regularity and Harnack's inequality for bounded weak solutions were established in [12, 13, 29, 39] and [22, 38], respectively. Besides, [28, 30, 35] are concerned with the asymptotic behavior of solutions of doubly nonlinear parabolic equations for certain values of the quantity $p+m$, and the local boundedness of the gradient was shown in [31] under the additional assumption that $u$ is strictly positive. Existence and uniqueness results for the Cauchy-Dirichlet problem with an inhomogeneity $\mu \in L^{\infty}\left(E_{T}, \mathbf{R}_{\geq 0}\right)$ were developed in [14, 15, 16] and generalized in [34], where $\mu \in L^{s}\left(E_{T}, \mathbf{R}_{\geq 0}\right)$ for some $s \equiv s(n, m, p)$. However, since the proof strategies are quite sophisticated due to the inherent difficulty of a double nonlinearity, there are relatively few achievements regarding doubly nonlinear parabolic equations, and, to 
the author's knowledge, there are no existence results for the corresponding measure data problem in the literature up to now, not even for the model equation (1.7). Nevertheless, certain special cases of (1.1) have been intensively studied (the survey [17] and its list of references offer a deeper insight for the interested reader). In particular, the equation passes into the porous medium equation when $p=2$, the $p$-Laplacian equation when $m=1$, and the well-known (linear) heat equation $\partial_{t} u=\Delta u$ can be obtained by simultaneously setting $p=2$ and $m=1$. For those equations, an enormous amount of mathematical literature is available, and it is impossible to list all proven outcomes. In order to get at least a better overview of the porous medium equation, one might especially appeal to $[1,5,6,8,10,26,27,36,37]$ and the lists of references therein. In addition, $[2,3,4,9,11,18,19,23]$ contain some famous accomplishments as regards $p$-Laplacian equations. Eventually, we should mention that the equation (1.7) is often displayed in the form $\omega^{1-p} \partial_{t} v^{\omega}-\operatorname{div}\left(|D v|^{p-2} D v\right)=\omega^{1-p} \mu$, which can be deduced from the former via the transformation $u=v^{\omega}$, where $\omega:=\frac{p-1}{p+m-2}$.

1.3. Notations. As to the notation, we always write $z=(x, t)$ for a point $z \in \mathbf{R}^{n+1} \cong \mathbf{R}^{n} \times \mathbf{R}$. As is customary, we denote by $\theta^{\prime}:=\frac{\theta}{\theta-1} \in[1, \infty]$ the Hölder conjugate of $\theta \in[1, \infty]$. Moreover, we use the abbreviations $U_{t}:=U \times(0, t)$ and $U_{t_{1}, t_{2}}:=U \times\left(t_{1}, t_{2}\right)$ for any $U \subset E, t \in(0, T)$ and $t_{1}, t_{2} \in[0, T]$ with $t_{1}<t_{2}$. $|U|$ marks the $n$-dimensional Lebesgue measure of the set $U$ (analogously, $\left|U_{t}\right|$ stands for the $(n+1)$-dimensional Lebesgue measure of $\left.U_{t}\right)$. By $\{u>\ell\}$, we express the superlevel set $\left\{(x, t) \in E_{T}: u(x, t)>\ell\right\}$ where the function $u$ exceeds the level $\ell>0$, and we address the positive part of $u$ as $u_{+}:=\max \{u, 0\}$. We denote the weak spatial derivative of the function $u$ by $D u=D_{x} u=\left(D_{x_{1}} u, D_{x_{2}} u, \ldots, D_{x_{n}} u\right)$, and $\partial_{t}=\frac{\partial}{\partial t}$ is the operator for the time derivative. Finally, by $\mathcal{M}^{+}\left(E_{T}\right)$, we mean the set of all non-negative Radon measures, and $c \equiv c(\cdot)$ stands for a constant, which may vary from line to line and depend only on the parameters in brackets.

1.4. The concept of very weak solutions. In this section, we will define our notion of a very weak solution. Throughout the whole article, we will abbreviate

$$
\alpha:=\frac{m-1}{p}
$$

in order to shorten the notation.

Definition 1.1. Let $\alpha$ as in (1.8). A non-negative function $u: E_{T} \rightarrow \mathbf{R}$ satisfying

$$
u \in L^{\infty}\left((0, T) ; L^{1}(E)\right), \quad u^{\alpha+1} \in L^{1}\left((0, T) ; W_{0}^{1,1}(E)\right)
$$

and the integrability property

$$
\mathbf{A}(\cdot, u, D u) \in L^{1}\left(E_{T}, \mathbf{R}^{n}\right)
$$

is termed a very weak solution of the Cauchy-Dirichlet problem (1.2) if and only if the identity

$$
\iint_{E_{T}}\left[-u \partial_{t} \varphi+\mathbf{A}(x, t, u, D u) \cdot D \varphi\right] d z=\iint_{E_{T}} \varphi d \mu
$$

holds true for any testing function $\varphi \in C^{1}\left(\overline{E_{T}}\right)$ vanishing on $[\partial E \times(0, T)] \cup[\bar{E} \times\{T\}]$. For very weak solutions $u$, the symbol $D u$ in (1.10) has to be understood in the sense of (1.11).

At this point, we have to give a meaning to the symbol $D u$ from the above definition and become aware of the sense in which it has to be understood. 
Remark 1.2. Since we have imposed $D u^{\alpha+1} \in L^{1}\left(E_{T}, \mathbf{R}^{n}\right)$, among others, as a condition on $u$, the existence of $D u$ cannot be assured in the previous definition. Formally, we set

$$
D u:=\frac{1}{\alpha+1} \chi_{\{u>0\}} u^{-\alpha} D u^{\alpha+1}
$$

and like to interpret $D u$ in that way. On $\{u>\sigma\}$, where $\sigma>0, D u$ indeed is the weak derivative of $u$, and we have $D u \in L^{1}\left(E_{T} \cap\{u>\sigma\}, \mathbf{R}^{n}\right)$.

For local results, the values of $u$ on the parabolic boundary are irrelevant such that we may speak of a solution of the equation (1.1) rather than of a solution of a Cauchy-Dirichlet problem. We specify this concept in the following definition.

Definition 1.3. We say that a non-negative function $u: E_{T} \rightarrow \mathbf{R}$ satisfying

$$
u \in L^{\infty}\left((0, T) ; L^{1}(E)\right), u^{\alpha+1} \in L^{1}\left((0, T) ; W^{1,1}(E)\right),
$$

and the integrability property (1.9) is a very weak solution of the doubly nonlinear parabolic equation (1.1) if and only if the identity (1.10) holds true for any testing function $\varphi \in C_{0}^{1}\left(E_{T}\right)$.

1.5. Main result and proof strategies. We now provide the main theorem of this paper, which concerns the existence of very weak solutions of the CauchyDirichlet problem (1.2). The proof of Theorem 1.4 will be performed in Chapter 4.

Theorem 1.4. Let $\mu \in \mathcal{M}^{+}\left(E_{T}\right)$, let $\alpha$ as in (1.8), and assume that the hypotheses (1.3)-(1.6) are in force. Then, there exists at least one very weak solution $u$ (in the sense of Definition 1.1) of the Cauchy-Dirichlet problem (1.2). Moreover, the solution fulfills $u^{\alpha+1} \in L^{q}\left((0, T) ; W^{1, q}(E)\right)$ for any $q$ satisfying $1 \leq q<p-\frac{n}{n(\alpha+1)+1}$.

In the following, we will outline the strategy of the proof of the above result. First of all, in Chapter 2, we will prepare some basic tools. In particular, we will quote a parabolic version of the Gagliardo-Nirenberg inequality as well as a lemma regarding weak derivatives of truncated functions, and introduce a time mollification procedure.

In Chapter 3, we will define our notion of a weak solution and ensure that, given a solution $u$, the function $\min \{u, \ell\}$ is a supersolution for $\ell>0$ (see Lemma 3.3). Considering supersolutions is motivated by the fact that one has to construct a bound for both the time and the spatial derivative of the functions from the approximating sequence $\left(u_{j}\right)_{j \in \mathbf{N}}$ in order to apply a compactness result from [32] in Subsection 4.3.3. However, only the spatial derivative $D u_{j}^{\alpha+1}$, but not the expression $D u_{j}$, is welldefined (see Remark 1.2). This conflict will be solved by truncating the functions $u_{j}$ from below by some number $\varepsilon>0$ such that the truncated functions $w_{j}^{(\varepsilon)}:=$ $\max \left\{u_{j}, \varepsilon\right\}$ stay away from zero and $D\left(w_{j}^{(\varepsilon)}\right)^{\alpha+1}$ can easily be converted to $D w_{j}^{(\varepsilon)}$. After rewriting $w_{j}^{(\varepsilon)}$ in terms of $\min \left\{u_{j}, \varepsilon\right\}$, the developed theory, especially the bound (3.15) for the time derivative of a supersolution, is applicable.

The fourth chapter is designated for the proof of the existence of a very weak solution of the Cauchy-Dirichlet problem (1.2). For that purpose, we will build regularized Cauchy-Dirichlet problems in (4.4), where the right-hand side measure $\mu \in \mathcal{M}^{+}\left(E_{T}\right)$ from (1.2) is replaced by a sequence $\left(\mu_{j}\right)_{j \in \mathbf{N}}$ of $L^{\infty}\left(E_{T}, \mathbf{R}_{\geq 0}\right)$-functions. The existence of weak solutions $\left(u_{j}\right)_{j \in \mathbf{N}}$ (see Definition 3.1) for these approximated problems follows from $[14,15]$. In Section 4.2 , we will deduce certain uniform a priori estimates for the approximating sequence $\left(u_{j}\right)_{j \in \mathbf{N}}$. In the general case of (1.2) comprising a Radon measure, we cannot expect to have a bound in $L^{p}\left(E_{T}, \mathbf{R}^{n}\right)$ for 
$\left(D u_{j}^{\alpha+1}\right)_{j \in \mathbf{N}}$. Instead, the key idea here is to establish an estimate for $\left(D u_{j}^{\alpha+1}\right)_{j \in \mathbf{N}}$ below the natural energy space, i.e. one attempts to find some exponent $1 \leq q<p$ which admits an $L^{q}\left(E_{T}, \mathbf{R}^{n}\right)$-bound for $D u_{j}^{\alpha+1}$ that is uniform with respect to $j \in \mathbf{N}$. Then, the uniform estimates will lead to the weak convergences (4.21) and (4.22), where it will be important on the one hand to show that $\left(u_{j}\right)_{j \in \mathbf{N}}$ subconverges not only weakly, but in fact strongly and almost everywhere, and, on the other hand to identify the weak limit of (a subsequence of) $\left(D u_{j}^{\alpha+1}\right)_{j \in \mathbf{N}}$ as $D u^{\alpha+1}$ (see Section 4.3). After that, we will pass to the limit in the equation, where the main difficulty will come from proving the pointwise convergence of the derivatives (4.69), which will subsequently grant the convergence of the integral involving the diffusion term.

\section{Preliminaries}

In this chapter, we will provide various tools, which will be needed in the existence proof.

2.1. Auxiliary lemmata. To begin with, we cite a parabolic Sobolev embedding (cf. [9, Prop. 3.1, p. 7]), which we will employ later in Section 4.2.

Lemma 2.1. Let $1<q<\infty$ and $0<r<\infty$. Then, there exists a constant $c \equiv c(n, q, r)$ such that for every $u \in L^{\infty}\left((0, T) ; L^{r}(E)\right) \cap L^{q}\left((0, T) ; W_{0}^{1, q}(E)\right)$ there holds the Gagliardo-Nirenberg inequality

$$
\iint_{E_{T}}|u|^{\ell} d z \leq c\left[\sup _{t \in(0, T)} \int_{E \times\{t\}}|u|^{r} d x\right]^{\frac{q}{n}} \iint_{E_{T}}|D u|^{q} d z,
$$

where $\ell$ is given by $\ell=\frac{q(n+r)}{n}$.

The following result, devoted to the derivation of truncated functions, can be retrieved from [14, p. 2736] (see also [15, Prop. 5.2] and [5, Lemma 2.2]).

Lemma 2.2. Let $1<q<\infty, \delta>0$, and suppose that $u: E_{T} \rightarrow \mathbf{R}$ is a nonnegative function such that $u^{\sigma} \in L^{q}\left((0, T) ; W^{1, q}(E)\right)$ for some $\sigma>1$. Then, $u^{(\delta)}:=$ $\max \{u, \delta\}$ has a weak derivative $D u^{(\delta)} \in L^{q}\left(E_{T}, \mathbf{R}^{n}\right)$, and $D u^{(\delta)}=\chi_{E_{T} \cap\{u>\delta\}} D u$, where $D u$ is defined by

$$
D u:=\frac{1}{\sigma} \chi_{E_{T} \cap\{u>0\}} u^{1-\sigma} D u^{\sigma} .
$$

Moreover, we have that

$$
\lim _{\delta \searrow 0}\left\|u^{\sigma-1} D u^{(\delta)}-\frac{1}{\sigma} D u^{\sigma}\right\|_{L^{q}\left(E_{T}, \mathbf{R}^{n}\right)}=0 .
$$

If additionally $u \geq \widetilde{\delta}$ on $E_{T}$ for some $\widetilde{\delta} \in(0, \delta)$, i.e. $u$ stays away from 0 , then $u_{(\delta)}:=\min \{u, \delta\}$ analogously has a weak derivative $D u_{(\delta)} \in L^{q}\left(E_{T}, \mathbf{R}^{n}\right)$, and $D u_{(\delta)}=$ $\chi_{E_{T} \cap\{u<\delta\}} D u$, where $D u$ is again defined by the formula in (2.2).

Next, we will define the auxiliary function $G_{\nu}$. It will turn up in the proof of Lemma 4.2, where we will need to use the property (2.3) from the following lemma. The corresponding proof can be found in [5, Lemma 2.3].

Lemma 2.3. For $\nu \in(0,1)$, we define the function $G_{\nu}:[0, \infty) \rightarrow \mathbf{R}$ by

$$
G_{\nu}(s):=\int_{0}^{s}\left[1-(1+\sigma)^{-\nu}\right] d \sigma=s-\frac{1}{1-\nu}\left[(1+s)^{1-\nu}-1\right]
$$

for any $s \geq 0$. Then, for any $\delta \in(0,1]$ and $s \geq 0$, there holds

$$
s \leq \delta+c_{\delta} G_{\nu}(s)
$$


for a constant $c_{\delta} \equiv \frac{c(\nu)}{\delta}$.

2.2. Mollification in time. We will now introduce a mollification in time and on its basis develop the regularized version (2.4) of the weak formulation (1.10).

Definition 2.4. For $v \in L^{1}\left(E_{T}\right)$, we define the mollification in time by

$$
\llbracket v \rrbracket_{h}(\cdot, t):=\frac{1}{h} \int_{0}^{t} e^{\frac{s-t}{h}} v(\cdot, s) d s
$$

and its time reversed analogue by

$$
\llbracket v \rrbracket_{h}(\cdot, t):=\frac{1}{h} \int_{t}^{T} e^{\frac{t-s}{h}} v(\cdot, s) d s
$$

for any $h \in(0, T]$ and $t \in[0, T]$. Likewise, one can define the time regularization of a vector-valued function $v^{\prime} \in L^{1}\left(E_{T}, \mathbf{R}^{n}\right)$.

For the main properties of this mollification, we refer to [7, Appendix B] and [21, Lemma 2.2]. One can now derive the regularized variant (2.4) of the weak formulation (1.10) (see [5, p. 3293] or [33, Thm. 2.10]). The time mollification procedure from Definition 2.4 allows us to insert in (2.4) testing functions whose time derivative does not necessarily have to exist. In other words, Lemma 2.5 admits testing functions containing the solution $u$ itself, avoiding an appearance of the quantity $\partial_{t} u$.

Lemma 2.5. If $u: E_{T} \rightarrow \mathbf{R}_{\geq 0}$ is a weak solution of the inhomogeneous doubly nonlinear parabolic equation (1.1) in the sense of Definition 3.1, then, its time mollification $\llbracket u \rrbracket_{h}$ fulfills the averaged equation

$$
\iint_{E_{T}}\left[\partial_{t} \llbracket u \rrbracket_{h} \varphi+\llbracket \mathbf{A}(x, t, u, D u) \rrbracket_{h} \cdot D \varphi\right] d z=\iint_{E_{T}} \llbracket \varphi \rrbracket_{h} d \mu
$$

of (1.10) for any testing function $\varphi \in C^{\infty}\left(\overline{E_{T}}\right)$ with compact support in $E_{T}$.

\section{Weak solutions and supersolutions}

In this chapter, we will examine weak solutions of the doubly nonlinear parabolic equation (1.1). This concept will be employed for the purpose of achieving the existence of a solution of regularized Cauchy-Dirichlet problems in Section 4.1. The central aim of this paragraph is establishing the bound (3.15) for the time derivative of a weak supersolution, which will follow from the Caccioppoli estimate from Lemma 3.4 and the measure bound (3.12). The necessity of studying supersolutions stems from the fact that we have to deal with truncated weak solutions in the existence proof. According to Lemma 3.3, the truncated function is no longer a weak solution, but a supersolution.

Definition 3.1. Let $\alpha$ as in (1.8). A non-negative function $u: E_{T} \rightarrow \mathbf{R}$ satisfying

$$
u \in C^{0}\left([0, T] ; L^{2}(E)\right) \quad \text { and } \quad u^{\alpha+1} \in L^{p}\left((0, T) ; W_{0}^{1, p}(E)\right)
$$

is termed a weak solution of the Cauchy-Dirichlet problem (1.2) if and only if the identity (1.10) holds true for any testing function $\varphi \in C^{1}\left(\overline{E_{T}}\right)$ vanishing on $[\partial E \times$ $(0, T)] \cup[\bar{E} \times\{T\}]$; cf. also Remark 1.2. What is more, analogous to Definition 1.3, we say that a non-negative function $u: E_{T} \rightarrow \mathbf{R}$ satisfying

$$
u \in C^{0}\left([0, T] ; L^{2}(E)\right) \quad \text { and } \quad u^{\alpha+1} \in L^{p}\left((0, T) ; W^{1, p}(E)\right)
$$


is a weak solution of the doubly nonlinear parabolic equation (1.1) if and only if the identity (1.10) holds true for any testing function $\varphi \in C_{0}^{1}\left(E_{T}\right)$.

Remark 3.2. Note that the existence of a weak solution of (1.2) cannot be guaranteed as long as the Cauchy-Dirichlet problem involving a general Radon measure $\mu \in \mathcal{M}^{+}\left(E_{T}\right)$ without any further qualities is considered. If actually $\mu \in L^{\gamma}\left(E_{T}, \mathbf{R}_{\geq 0}\right)$ for $\gamma$ as in (4.18), one can prove the existence of weak solutions (see Remark 4.3). The prerequisite in the previous definition that $u$ belongs to the space $C^{0}\left([0, T] ; L^{2}(E)\right)$ originates from the classical existence theorems for $p$-Laplacian equations in [24, 25], and the requirement $u^{\alpha+1} \in L^{p}\left((0, T) ; W^{1, p}(E)\right)$ is in perfect accordance with $[5$, cond. (1.5)] or the case $m>1$ of [10, p. 34] since $\alpha+1=\frac{m+1}{2}$ for $p=2$. Another approach can be found, for instance, in [15, 28, 30, 38, 39], where the regularity properties (3.1) are replaced by the conditions

$$
u \in C^{0}\left([0, T] ; L^{\beta+2}(E)\right) \quad \text { and } \quad u^{\beta+1} \in L^{p}\left((0, T) ; W^{1, p}(E)\right)
$$

for $\beta:=\frac{m-1}{p-1}$. Provided that $u \in L^{\infty}\left(E_{T}\right)$, one can infer that $D u^{\alpha+1} \in L^{p}\left(E_{T}, \mathbf{R}^{n}\right)$ implies $D u^{\beta+1} \in L^{p}\left(E_{T}, \mathbf{R}^{n}\right)$ and both representations $\frac{1}{\alpha+1} \chi_{\{u>0\}} u^{-\alpha} D u^{\alpha+1}$ and $\frac{1}{\beta+1} \chi_{\{u>0\}} u^{-\beta} D u^{\beta+1}$ for $D u$ coincide (see [16, p. 787]). On the contrary, under the assumption that $D u^{\beta+1} \in L^{p}\left(E_{T}, \mathbf{R}^{n}\right)$, there holds $D u^{\alpha+1} \in L^{p}\left(E_{T}, \mathbf{R}^{n}\right)$ only as long as one knows a lower bound $u \geq \sigma$ for some $\sigma>0$.

We now start considering weak (super-)solutions. The following lemma ensures that, for a weak solution $u$ and a given $\ell>0$, the function $W_{\ell}(u):=\min \{u, \ell\}$ is a weak supersolution. In the case $p=2$, this result can be retrieved from $[6$, Lemma 3.1], where the conditions (3.2) were employed in the definition of a weak solution. However, replacing the testing function from [6, Lemma 3.1] by $\varphi \frac{\ell-W_{\ell}(u)}{\varrho+\ell-W_{\ell}(u)}$ with a fixed $\varrho>0, \ell>0$ and a non-negative function $\varphi \in C_{0}^{1}\left(E_{T}\right)$, the proof works with only minor modifications when using our regularity assumptions (3.1) instead.

Lemma 3.3. Suppose that $u: E_{T} \rightarrow \mathbf{R}$ is a weak solution of the doubly nonlinear parabolic equation (1.1) under the structure conditions (1.3) and (1.4) and with an inhomogeneity $\mu \in L^{1}\left(E_{T}, \mathbf{R}_{\geq 0}\right)$. Furthermore, assume that for some $\ell>0$, the truncation $W_{\ell}(u):=\min \{u, \ell\}$ satisfies $D W_{\ell}(u)^{\alpha+1} \in L^{p}\left(E_{T}, \mathbf{R}^{n}\right)$. Then, $W_{\ell}(u)$ is a weak supersolution of (1.1), i.e. we have

$$
\iint_{E_{T}}\left[-W_{\ell}(u) \partial_{t} \varphi+\mathbf{A}\left(x, t, W_{\ell}(u), D W_{\ell}(u)\right) \cdot D \varphi\right] d z \geq 0
$$

for any non-negative $\varphi \in C_{0}^{1}\left(E_{T}\right)$.

In the sequel, we deal with bounded weak supersolutions $w: E_{T} \rightarrow \mathbf{R}$ of the doubly nonlinear parabolic equation (1.1), i.e. the non-negative function $w$ fulfills

$$
w \in C^{0}\left([0, T] ; L^{2}(E)\right), \quad w^{\alpha+1} \in L^{p}\left((0, T) ; W^{1, p}(E)\right)
$$

as well as the inequality

$$
\iint_{E_{T}}\left[-w \partial_{t} \varphi+\mathbf{A}(x, t, w, D w) \cdot D \varphi\right] d z \geq 0
$$

for any non-negative $\varphi \in C_{0}^{1}\left(E_{T}\right)$. First, we will establish the following Caccioppoli type inequality (see [5, Lemma 5.2] and [20, Lemma 2.15] for the porous medium equation case). 
Lemma 3.4. Let $w: E_{T} \rightarrow \mathbf{R}$ as in (3.4) be a bounded weak supersolution of the doubly nonlinear parabolic equation (1.1) in the sense that (3.5) is valid. Moreover, suppose that $0 \leq w \leq M$ for some $M>0$, and the hypotheses (1.3) and (1.4) are in force. Then, for any $0 \leq t_{1}<t_{2} \leq T$, the Caccioppoli type estimate

$$
\iint_{E_{t_{1}, t_{2}}} \eta^{p}\left|D w^{\alpha+1}\right|^{p} d z \leq c\left[M^{p+m-1}\left(t_{2}-t_{1}\right) \int_{E}|D \eta|^{p} d x+M^{2} \int_{E} \eta^{p} d x\right]
$$

holds true for any non-negative $\eta \in C_{0}^{1}(E)$ with a constant $c \equiv c\left(m, p, C_{0}, C_{1}\right)$.

Proof. We define $w^{(\kappa)}:=\max \{w, \kappa\}$ for $0<\kappa<M$ and choose in (3.5) the testing function $\varphi:=\eta^{p} \zeta\left(M-w^{(\kappa)}\right)$, where $\eta \in C_{0}^{1}(E)$ is non-negative and $\zeta \in$ $W_{0}^{1, \infty}(\mathbf{R},[0,1])$ satisfies

$$
\zeta(t):= \begin{cases}1 & \text { for } t \in\left[t_{1}, t_{2}\right], \\ 0 & \text { for } t \in \mathbf{R} \backslash\left(t_{1}-\tau, t_{2}+\tau\right), \\ \frac{1}{\tau}\left(t-t_{1}+\tau\right) & \text { for } t \in\left(t_{1}-\tau, t_{1}\right), \\ -\frac{1}{\tau}\left(t-t_{2}-\tau\right) & \text { for } t \in\left(t_{2}, t_{2}+\tau\right)\end{cases}
$$

with $0<\tau<\min \left\{t_{1}, T-t_{2}\right\}$. As a result, (3.5) reads as

$$
\begin{aligned}
\mathcal{I}_{\tau}^{(1)}:= & \iint_{E_{T}} \eta^{p} \zeta \mathbf{A}(x, t, w, D w) \cdot D w^{(\kappa)} d z \\
\leq & p \iint_{E_{T}} \eta^{p-1} \zeta\left(M-w^{(\kappa)}\right) \mathbf{A}(x, t, w, D w) \cdot D \eta d z \\
& -\iint_{E_{T}} \eta^{p} w\left(M-w^{(\kappa)}\right) \partial_{t} \zeta d z+\iint_{E_{T}} \eta^{p} \zeta w \partial_{t} w^{(\kappa)} d z \\
= & : \mathcal{I}_{\tau}^{(2)}+\mathcal{I}_{\tau}^{(3)}+\mathcal{I}_{\tau}^{(4)} .
\end{aligned}
$$

Treating the integrals containing time derivatives, we reason only formally. The argument can be made rigorous by exerting the mollification from Definition 2.4. We start with the term $\mathcal{I}_{\tau}^{(3)}$ and work with the definition of $\zeta$ and the facts that $w \leq M$ and $w^{(\kappa)} \geq 0$ to receive

$$
\mathcal{I}_{\tau}^{(3)} \leq \int_{t_{2}}^{t_{2}+\tau}\left|\partial_{t} \zeta\right| \int_{E} \eta^{p} w\left(M-w^{(\kappa)}\right) d x d t \leq M^{2} \int_{E} \eta^{p} d x
$$

For the integral $\mathcal{I}_{\tau}^{(4)}$, we use the identity $w \partial_{t} w^{(\kappa)}=w^{(\kappa)} \partial_{t} w^{(\kappa)}=\frac{1}{2} \partial_{t}\left|w^{(\kappa)}\right|^{2}$, an integration by parts, and the inequality $w^{(\kappa)} \leq M$. In this way, we find

$$
\mathcal{I}_{\tau}^{(4)}=-\frac{1}{2} \iint_{E_{T}} \eta^{p}\left|w^{(\kappa)}\right|^{2} \partial_{t} \zeta d z \leq \frac{1}{2} \int_{t_{2}}^{t_{2}+\tau}\left|\partial_{t} \zeta\right| \int_{E} \eta^{p}\left|w^{(\kappa)}\right|^{2} d x d t \leq \frac{1}{2} M^{2} \int_{E} \eta^{p} d x .
$$

Next, applying the growth condition (1.4), the assumption $w \leq M$, and Young's inequality, we obtain

$$
\begin{aligned}
\lim _{\tau \searrow 0} \mathcal{I}_{\tau}^{(2)} & \leq c \lim _{\tau \searrow 0} \iint_{E_{T}} \eta^{p-1} \zeta M w^{m-1}|D w|^{p-1}|D \eta| d z \\
& \leq c \iint_{E_{t_{1}, t_{2}}} \eta^{p-1} M^{\alpha+1}\left|D w^{\alpha+1}\right|^{p-1}|D \eta| d z \\
& \leq \delta \iint_{E_{t_{1}, t_{2}}} \eta^{p}\left|D w^{\alpha+1}\right|^{p} d z+c_{\delta} M^{p+m-1}\left(t_{2}-t_{1}\right) \int_{E}|D \eta|^{p} d x
\end{aligned}
$$


for any $\delta \in(0,1)$ with a constant $c_{\delta} \equiv c_{\delta}\left(m, p, C_{1}, \delta\right)$. Finally, from Lemma 2.2, we know that $D w^{(\kappa)}=\chi_{\{w>\kappa\}} D w$ such that, inserting the ellipticity condition (1.3), we can estimate the integral $\mathcal{I}_{\tau}^{(1)}$ from below:

$$
\begin{aligned}
\lim _{\tau \searrow 0} \mathcal{I}_{\tau}^{(1)} & =\lim _{\tau \searrow 0} \iint_{E_{T}} \chi_{\{w>\kappa\}} \eta^{p} \zeta \mathbf{A}(x, t, w, D w) \cdot D w d z \\
& \geq C_{0} \iint_{E_{t_{1}, t_{2}}} \chi_{\{w>\kappa\}} \eta^{p} w^{m-1}|D w|^{p} d z=\bar{c} \iint_{E_{t_{1}, t_{2}}} \chi_{\{w>\kappa\}} \eta^{p}\left|D w^{\alpha+1}\right|^{p} d z,
\end{aligned}
$$

where $\bar{c} \equiv \bar{c}\left(m, p, C_{0}\right)$ is a constant. Passing to the limits $\tau \searrow 0$ and $\kappa \searrow 0$, joining the estimates for $\mathcal{I}_{\tau}^{(1)}-\mathcal{I}_{\tau}^{(4)}$ with (3.8), and selecting $\delta:=\bar{c} / 2$, we can reabsorb the energy term appearing on the right-hand side into the left and conclude that (3.6) is valid for any choice of $0<t_{1}<t_{2}<T$. This implies the claim for any $0 \leq t_{1}<t_{2} \leq T$.

Remark 3.5. Consider a non-negative weak supersolution $w$ of the doubly nonlinear parabolic equation (1.1). Then, by the Riesz representation theorem, there exists a non-negative Radon measure $\mu_{w}$ such that there holds

$$
\iint_{E_{T}}\left[-w \partial_{t} \varphi+\mathbf{A}(x, t, w, D w) \cdot D \varphi\right] d z=\iint_{E_{T}} \varphi d \mu_{w}
$$

for any $\varphi \in C_{0}^{1}\left(E_{T}\right)$.

In the following lemma, we will derive a local estimate for the corresponding measure $\mu_{w}$ to a bounded weak supersolution $w$.

Lemma 3.6. Let $w: E_{T} \rightarrow \mathbf{R}$ as in (3.4), (3.5) be a bounded weak supersolution of the equation (1.1) under the structure conditions (1.3) and (1.4). Besides, suppose that $0 \leq w \leq M$ for some $M>0$, and let $\mu_{w}$ be the associated Radon measure according to Remark 3.5. Then, for any $U \Subset E$, any $0<t_{1}<t_{2}<T$, and any $\eta \in C_{0}^{1}(E,[0,1])$ with $\eta \equiv 1$ on $U$, we have

$$
\mu_{w}\left(U_{t_{1}, t_{2}}\right) \leq c\left[M^{p+m-2}\left(t_{2}-t_{1}\right) \int_{E}|D \eta|^{p} d x+M \int_{E} \eta^{p} d x\right]
$$

with a constant $c \equiv c\left(m, p, C_{0}, C_{1}\right)$.

Proof. Let $\eta \in C_{0}^{1}(E,[0,1])$ be as in the statement of the lemma and choose the cut-off function in time $\zeta$ as in (3.7). Then, testing (3.11) with $\varphi:=\eta^{p} \zeta$, we get

$$
\begin{aligned}
\mu_{w}\left(U_{t_{1}, t_{2}}\right) & =\iint_{U_{t_{1}, t_{2}}} \eta^{p} \zeta d \mu_{w} \leq \iint_{E_{T}} \eta^{p} \zeta d \mu_{w} \\
& =-\iint_{E_{T}} \eta^{p} w \partial_{t} \zeta d z+p \iint_{E_{T}} \eta^{p-1} \zeta \mathbf{A}(x, t, w, D w) \cdot D \eta d z \\
& =: \mathcal{J}^{(1)}+\mathcal{J}_{\tau}^{(2)} .
\end{aligned}
$$

With the same arguments as in (3.9), the first integral simplifies to

$$
\mathcal{J}^{(1)} \leq-\int_{t_{2}}^{t_{2}+\tau} \partial_{t} \zeta \int_{E} \eta^{p} w d x d t \leq M \int_{E} \eta^{p} d x .
$$


Next, by the growth condition (1.4), the calculations performed in (3.10), and the Caccioppoli inequality (3.6), we infer

$$
\begin{aligned}
\lim _{\tau \searrow 0} \mathcal{J}_{\tau}^{(2)} & \leq c \lim _{\tau \searrow 0} \iint_{E_{T}} \eta^{p-1} \zeta w^{m-1}|D w|^{p-1}|D \eta| d z \\
& \leq c M^{-1} \iint_{E_{t_{1}, t_{2}}} \eta^{p}\left|D w^{\alpha+1}\right|^{p} d z+c M^{p+m-2}\left(t_{2}-t_{1}\right) \int_{E}|D \eta|^{p} d x \\
& \leq c\left[M^{p+m-2}\left(t_{2}-t_{1}\right) \int_{E}|D \eta|^{p} d x+M \int_{E} \eta^{p} d x\right]
\end{aligned}
$$

with a constant $c \equiv c\left(m, p, C_{0}, C_{1}\right)$. Combining the last two estimates with (3.13), we conclude that (3.12) holds true.

Corollary 3.7. Let $w: E_{T} \rightarrow \mathbf{R}$ as in (3.4), (3.5) be a bounded weak supersolution of the equation (1.1) under the structure conditions (1.3) and (1.4). Besides, suppose that $0 \leq w \leq M$ for some $M>0$, and let $\mu_{w}$ be the associated Radon measure according to Remark 3.5. Then, for any pair of sets $U \Subset W \Subset E$ and any $0<t_{1}<t_{2}<T$, the following local energy and measure bound holds true with a constant $c \equiv c\left(m, p, C_{0}, C_{1}\right)$ :

$$
\iint_{U_{t_{1}, t_{2}}}\left|D w^{\alpha+1}\right|^{p} d z+M \mu_{w}\left(U_{t_{1}, t_{2}}\right) \leq c M|W|\left[\frac{M^{p+m-2}\left(t_{2}-t_{1}\right)}{\operatorname{dist}(U, \partial W)^{p}}+M\right] .
$$

Proof. Let $\eta \in C_{0}^{1}(E,[0,1])$ with $\eta \equiv 1$ on $U, \eta \equiv 0$ on $E \backslash W$, and $|D \eta| \leq$ $\frac{2}{\operatorname{dist}(U, \partial W)}$. Then, noting that $\operatorname{spt}(\eta) \subset W$, the estimate (3.14) is an immediate consequence of Lemma 3.4 and Lemma 3.6.

Having at hand the bound (3.14), we can take advantage of Remark 3.5 to deduce a local bound for the time derivative of $w$ in the next lemma.

Lemma 3.8. Let $w: E_{T} \rightarrow \mathbf{R}$ as in (3.4), (3.5) be a bounded weak supersolution of the equation (1.1) under the structure conditions (1.3) and (1.4). Provided that $0 \leq w \leq M$ for some $M>0$, we have

$$
\left\|\partial_{t} w\right\|_{L^{1}\left(\left(t_{1}, t_{2}\right) ; W^{-1,1}(U)\right)} \leq c|W|\left[\frac{M^{p+m-2}\left(t_{2}-t_{1}\right)}{\operatorname{dist}(U, \partial W)^{p}}+M\right]
$$

for any pair of sets $U \Subset W \Subset E$ with $\operatorname{dist}(U, \partial W) \leq 1$ and any $0<t_{1}<t_{2}<T$, where $c \equiv c\left(m, p, C_{0}, C_{1}\right)$ is a constant.

Proof. For the proof, we fix $U \Subset W \Subset E$ with $\operatorname{dist}(U, \partial W) \leq 1$ and $0<t_{1}<t_{2}<$ $T$. Then, we consider (3.11) for functions $\varphi \in C_{0}^{1}\left(E_{T}\right)$ with compact support in $U_{t_{1}, t_{2}}$ and $\|\varphi\|_{L^{\infty}\left(\left(t_{1}, t_{2}\right) ; W^{1, \infty}(U)\right)} \leq 1$. Using in turn the growth condition (1.4), Hölder's inequality, the assumption $w \leq M$, Young's inequality, and finally the bound (3.14), we obtain

$$
\begin{aligned}
\left|\iint_{E_{T}} w \partial_{t} \varphi d z\right| & \leq\left|\iint_{E_{T}} \varphi d \mu_{w}\right|+\left|\iint_{E_{T}} \mathbf{A}(x, t, w, D w) \cdot D \varphi d z\right| \\
& \leq \mu_{w}\left(U_{t_{1}, t_{2}}\right)+C_{1} \iint_{U_{t_{1}, t_{2}}} w^{m-1}|D w|^{p-1}|D \varphi| d z \\
& \leq \mu_{w}\left(U_{t_{1}, t_{2}}\right)+C_{1}\left|U_{t_{1}, t_{2}}\right|^{\frac{1}{p}}\left[\iint_{U_{t_{1}, t_{2}}} w^{\alpha p p^{\prime}}|D w|^{p} d z\right]^{\frac{1}{p^{\prime}}}
\end{aligned}
$$




$$
\begin{aligned}
& \leq \mu_{w}\left(U_{t_{1}, t_{2}}\right)+c\left|U_{t_{1}, t_{2}}\right|^{\frac{1}{p}} M^{\alpha}\left[\iint_{U_{t_{1}, t_{2}}}\left|D w^{\alpha+1}\right|^{p} d z\right]^{\frac{1}{p^{\prime}}} \\
& \leq \mu_{w}\left(U_{t_{1}, t_{2}}\right)+c M^{-1} \iint_{U_{t_{1}, t_{2}}}\left|D w^{\alpha+1}\right|^{p} d z+c M^{p+m-2}\left|U_{t_{1}, t_{2}}\right| \\
& \leq c|W|\left[\frac{M^{p+m-2}\left(t_{2}-t_{1}\right)}{\operatorname{dist}(U, \partial W)^{p}}+M\right]+c M^{p+m-2}|W|\left(t_{2}-t_{1}\right)
\end{aligned}
$$

with a constant $c \equiv c\left(m, p, C_{0}, C_{1}\right)$. Since $\operatorname{dist}(U, \partial W) \leq 1$, taking the supremum over all $\varphi \in C_{0}^{1}\left(U_{t_{1}, t_{2}}\right)$ with $\|\varphi\|_{L^{\infty}\left(\left(t_{1}, t_{2}\right) ; W^{1, \infty}(U)\right)} \leq 1$ yields the claim.

\section{Existence of very weak solutions: The proof of Theorem 1.4}

Proof of Theorem 1.4. In this chapter, we will perform the proof of Theorem 1.4. We will proceed as described in Section 1.5.

4.1. The approximation scheme. Let $\left(\mu_{j}\right)_{j \in \mathbf{N}} \subset L^{\infty}\left(E_{T}, \mathbf{R}_{\geq 0}\right)$ be a sequence of non-negative bounded functions approximating the inhomogeneity $\mu \in \mathcal{M}^{+}\left(E_{T}\right)$ of the Cauchy-Dirichlet problem (1.2) in the sense that $\left(\mu_{j}\right)_{j \in \mathbf{N}}$ fulfills

$$
\mu_{j}\left(E_{T}\right):=\iint_{E_{T}} \mu_{j} d z \leq \mu\left(E_{T}\right)<\infty
$$

for any $j \in \mathbf{N}$, and the associated measures $\mu_{j} d z$ converge to $\mu$ weakly* in $\mathcal{M}^{+}\left(E_{T}\right)$ as $j \rightarrow \infty$, i.e. there holds

$$
\lim _{j \rightarrow \infty} \iint_{E_{T}} \mu_{j} \varphi d z=\iint_{E_{T}} \varphi d \mu
$$

for any $\varphi \in C_{0}^{0}\left(E_{T}\right)$. In what follows, we consider for $j \in \mathbf{N}$ the regularized equations

$$
\partial_{t} u_{j}-\operatorname{div}\left(\mathbf{A}\left(x, t, u_{j}, D u_{j}\right)\right)=\mu_{j}
$$

and the corresponding Cauchy-Dirichlet problems

$$
\begin{cases}\partial_{t} u_{j}-\operatorname{div}\left(\mathbf{A}\left(x, t, u_{j}, D u_{j}\right)\right)=\mu_{j} & \text { in } E_{T}, \\ u_{j}=0 & \text { on } \Gamma_{T},\end{cases}
$$

respectively. They admit a weak solution (see Definition 3.1) by the following existence theorem, which can be retrieved from [14, Thm. 3.1] in the case $m \geq 1, p \geq 2$, and from [15, Thm. 1.1] for $m \geq 1, p \in\left(\frac{2 n}{n+2}, 2\right)$. We notice that the Lipschitz condition $[15,(L)$, p. 847] is only required for uniqueness matters such that we do not need to impose an assumption of this type on the vector field $\mathbf{A}$ here. Moreover, note that the condition $[14,(d)$, p. 2725] is a consequence of (1.4) in our context.

Lemma 4.1. Let $j \in \mathbf{N}, \mu_{j} \in L^{\infty}\left(E_{T}, \mathbf{R}_{\geq 0}\right)$, let $\alpha$ as in (1.8), and assume that the hypotheses (1.3), (1.4), and (1.6) are in force. Then, there exists at least one non-negative weak solution $u_{j}$ of the Cauchy-Dirichlet problem (4.4) satisfying $u_{j} \in C^{0}\left([0, T] ; L^{2}(E)\right), u_{j}^{\alpha+1} \in L^{p}\left((0, T) ; W_{0}^{1, p}(E)\right)$, and

$$
\iint_{E_{T}}\left[-u_{j} \partial_{t} \varphi+\mathbf{A}\left(x, t, u_{j}, D u_{j}\right) \cdot D \varphi\right] d z=\iint_{E_{T}} \mu_{j} \varphi d z
$$


for any testing function $\varphi \in C^{1}\left(\overline{E_{T}}\right)$ vanishing on $[\partial E \times(0, T)] \cup[\bar{E} \times\{T\}]$. Note that the symbol $D u_{j}$ has to be understood analogous to (1.11), i.e.

$$
D u_{j}:=\frac{1}{\alpha+1} \chi_{\left\{u_{j}>0\right\}} u_{j}^{-\alpha} D u_{j}^{\alpha+1} .
$$

4.2. Energy estimates below the natural growth. In this section, we will prove certain a priori estimates for the weak solutions $u_{j}(j \in \mathbf{N})$ of the CauchyDirichlet problems (4.4) from Lemma 4.1. In view of the presence of the Radon measure $\mu$ on the right-hand side of the considered equation (1.1), choosing $\varphi \sim u_{j}$ as a testing function does not lead to energy bounds independent of $j$ since, for the approximating sequence $\left(\mu_{j}\right)_{j \in \mathbf{N}}$, only the uniform $L^{1}\left(E_{T}\right)$-bound (4.1) is available (see also Remark 4.3). Therefore, we have to design our testing function in a more subtle way in order to accomplish the following estimate below the natural energy space. We remark that a similar testing function was already constructed in [19, p. 148] for elliptic $p$-Laplacian equations.

Lemma 4.2. Let $\mu \in \mathcal{M}^{+}\left(E_{T}\right)$ be the non-negative Radon measure from (1.2), suppose that the structure conditions (1.3)-(1.6) hold, and let $\alpha$ as in (1.8). Moreover, let $q$ such that

$$
1 \leq q<p-\frac{n}{n(\alpha+1)+1}
$$

and let $u_{j}$ be the weak solution of the Cauchy-Dirichlet problem (4.4) with $\mu_{j} \in$ $L^{\infty}\left(E_{T}, \mathbf{R}_{\geq 0}\right)$ fulfilling (4.1) and (4.2), where $j \in \mathbf{N}$. Then, the estimates

$$
\sup _{t \in(0, T)} \int_{E \times\{t\}} u_{j} d x \leq c<\infty,
$$

$$
\iint_{E_{T}}\left|D u_{j}^{\alpha+1}\right|^{q} d z \leq c<\infty
$$

and

$$
\iint_{E_{T}} u_{j}^{\left[\alpha+1+\frac{1}{n}\right] q} d z \leq c<\infty
$$

hold with a constant $c \equiv c\left(n, m, p, q, C_{0},\left|E_{T}\right|, \mu\left(E_{T}\right)\right)$. In particular, $c$ does not depend on $j$.

Proof. Let $\nu \in(0,1)$ and $\varepsilon>0$, and define the testing function

$$
\varphi:=\zeta\left(1-\frac{1}{\left[1+\left(u_{j}-\varepsilon\right)_{+}\right]^{\nu}}\right)
$$

where the cut-off function in time $\zeta \in W^{1, \infty}(\mathbf{R},[0,1])$ is given by

$$
\zeta(t):= \begin{cases}1 & \text { for } t \in(-\infty, \tau-\delta], \\ -\frac{1}{\delta}(t-\tau) & \text { for } t \in(\tau-\delta, \tau), \\ 0 & \text { for } t \in[\tau, \infty)\end{cases}
$$

for a fixed $\tau \in(0, T)$ and $\delta \in(0, \tau)$. Now, note that $\varphi=0$ on $\partial E_{T}$ and plug $\varphi$ in the weak formulation (4.5) of the Cauchy-Dirichlet problem (4.4). As before, the reasoning can be made rigorous by applying the mollification in time introduced in Definition 2.4, however, for the sake of brevity, we only argue formally concerning the use of time derivatives. In the following, we will analyze all integrals appearing in the weak form. For the first term, employing the facts that $\partial_{t}\left(u_{j}-\varepsilon\right)_{+}=\chi_{\left\{u_{j}>\varepsilon\right\}} \partial_{t} u_{j}$ 
and $\varphi=0$ on $\left\{u_{j} \leq \varepsilon\right\}$, and subsequently an integration by parts as well as the Lebesgue differentiation theorem, we obtain

$$
\begin{aligned}
\iint_{E_{T}} \varphi \partial_{t} u_{j} d z & =\iint_{E_{T}} \zeta \partial_{t}\left[\int_{0}^{\left(u_{j}-\varepsilon\right)_{+}}\left[1-(1+s)^{-\nu}\right] d s\right] d z \\
& =\frac{1}{\delta} \iint_{E \times(\tau-\delta, \tau)} \int_{0}^{\left(u_{j}-\varepsilon\right)_{+}}\left[1-(1+s)^{-\nu}\right] d s d z \\
& \rightarrow \int_{E \times\{\tau\}} G_{\nu}\left(\left(u_{j}-\varepsilon\right)_{+}\right) d x
\end{aligned}
$$

as $\delta \searrow 0$ for almost every $\tau \in(0, T)$, where $G_{\nu}$ is defined in Lemma 2.3. Next, by Lemma 2.2, we know that

$$
D \varphi=\zeta \chi_{\left\{u_{j}>\varepsilon\right\}} \frac{\frac{\nu}{\alpha+1} u_{j}^{-\alpha} D u_{j}^{\alpha+1}}{\left[1+\left(u_{j}-\varepsilon\right)_{+}\right]^{\nu+1}}
$$

such that the diffusion term can be estimated with the help of the ellipticity condition (1.3) in the following way:

$$
\lim _{\delta \searrow 0} \iint_{E_{T}} \mathbf{A}\left(x, t, u_{j}, D u_{j}\right) \cdot D \varphi d z \geq c \iint_{E_{\tau}} \frac{\chi_{\left\{u_{j}>\varepsilon\right\}}\left|D u_{j}^{\alpha+1}\right|^{p}}{\left[1+\left(u_{j}-\varepsilon\right)_{+}\right]^{\nu+1}} d z,
$$

where $c \equiv c\left(m, p, C_{0}, \nu\right)$. Finally, regarding that $0 \leq \varphi \leq 1$ and using the bound (4.1), we can compute

$$
\iint_{E_{T}} \mu_{j} \varphi d z \leq \mu\left(E_{T}\right)
$$

for the third term. Thus, by taking the supremum over all $\tau \in(0, T)$, we infer from (4.5), (4.12)-(4.14) and (2.3) that

$$
\sup _{t \in(0, T)} \int_{E \times\{t\}}\left(u_{j}-\varepsilon\right)_{+} d x+\iint_{E_{T}} \frac{\chi_{\left\{u_{j}>\varepsilon\right\}}\left|D u_{j}^{\alpha+1}\right|^{p}}{\left[1+\left(u_{j}-\varepsilon\right)_{+}\right]^{\nu+1}} d z \leq c,
$$

and since $u_{j} \geq 0$ on $E_{T}$, the dominated convergence theorem yields

$$
\sup _{t \in(0, T)} \int_{E \times\{t\}} u_{j} d x+\iint_{E_{T}} \frac{\left|D u_{j}^{\alpha+1}\right|^{p}}{\left(1+u_{j}\right)^{\nu+1}} d z \leq c
$$

in the limit $\varepsilon \searrow 0$, where $c \equiv c\left(m, p, C_{0},|E|, \mu\left(E_{T}\right), \nu\right)$ is a constant. The preceding inequality proves (4.8). In order to establish the estimate (4.9), we first apply the Gagliardo-Nirenberg inequality (2.1) to $u_{j}^{\alpha+1}$, where we set $r:=\frac{1}{\alpha+1}$ and choose $\nu$ such that

$$
\frac{q(n+r)}{n}=\frac{q(\nu+1)}{(\alpha+1)(p-q)}
$$

Note that the latter identity is equivalent to

$$
q=p-\frac{n(\nu+1)}{n(\alpha+1)+1},
$$


and therefore, since we assumed that $q$ fulfills the smallness property (4.7), we can always find a number $\nu \in(0,1)$ such that (4.16) is satisfied. Lemma 2.1 shows

$$
\begin{aligned}
\iint_{E_{T}} u_{j}^{\frac{q(\nu+1)}{p-q}} d z & =\iint_{E_{T}}\left|u_{j}^{\alpha+1}\right|^{\frac{q(n+r)}{n}} d z \\
& \leq c\left[\sup _{t \in(0, T)} \int_{E \times\{t\}} u_{j} d x\right]^{\frac{q}{n}} \iint_{E_{T}}\left|D u_{j}^{\alpha+1}\right|^{q} d z \\
& \leq c \iint_{E_{T}}\left|D u_{j}^{\alpha+1}\right|^{q} d z
\end{aligned}
$$

with a constant $c \equiv c\left(n, m, p, q, C_{0},|E|, \mu\left(E_{T}\right)\right)$, where we inserted the estimate (4.8) in the last step. Next, we make use of Hölder's inequality, (4.15) and (4.17), and eventually Young's inequality to obtain that

$$
\begin{aligned}
\iint_{E_{T}}\left|D u_{j}^{\alpha+1}\right|^{q} d z & \leq\left[\iint_{E_{T}} \frac{\left|D u_{j}^{\alpha+1}\right|^{p}}{\left(1+u_{j}\right)^{\nu+1}} d z\right]^{\frac{q}{p}}\left[\iint_{E_{T}}\left(1+u_{j}\right)^{\frac{q(\nu+1)}{p-q}} d z\right]^{1-\frac{q}{p}} \\
& \leq c\left[\left|E_{T}\right|+\iint_{E_{T}} u_{j}^{\frac{q(\nu+1)}{p-q}} d z\right]^{1-\frac{q}{p}} \\
& \leq c+c\left[\iint_{E_{T}}\left|D u_{j}^{\alpha+1}\right|^{q} d z\right]^{1-\frac{q}{p}} \leq c+\frac{p-q}{p} \iint_{E_{T}}\left|D u_{j}^{\alpha+1}\right|^{q} d z
\end{aligned}
$$

with a constant $c \equiv c\left(n, m, p, q, C_{0},\left|E_{T}\right|, \mu\left(E_{T}\right)\right)$. Now, reabsorbing the right-hand side integral into the left, one concludes that (4.9) is valid, and (4.10) is a direct consequence of (4.17) and (4.9).

Remark 4.3. In the foregoing lemma, the necessity of the constraint (1.5) becomes apparent since a number $q$ as in (4.7) can only be found provided that (1.5) holds. The assumption is natural in the sense that it coincides with the well-known conditions for porous medium type and $p$-Laplacian measure data problems (see $[5$, cond. (6.7)] and [2, Rem. $1.5 \& 1.7]$, respectively). If actually $\mu \equiv f \in L^{\gamma}\left(E_{T}, \mathbf{R}_{\geq 0}\right)$ for

$$
\gamma:=1+\frac{n}{n(p+m-2)+2 p},
$$

one can retrieve an energy estimate of the form

$$
\sup _{t \in(0, T)} \int_{E \times\{t\}} u_{j}^{2} d x+\iint_{E_{T}}\left|D u_{j}^{\alpha+1}\right|^{p} d z \leq c<\infty
$$

with a constant $c \equiv c\left(n, m, p, C_{0},\left|E_{T}\right|,\|f\|_{L^{\gamma}\left(E_{T}\right)}\right)$ independent of $j$ by testing the weak formulation with $\varphi:=\zeta u_{j}$, where $\zeta$ is as in (4.11). Hence, in the case of an $L^{\gamma}\left(E_{T}\right)$-inhomogeneity $f$, the presence of a variable $q$ as in (4.7) is not required, which is why the restriction (1.5) can be dropped. In order to explain the exponent $\gamma$ from (4.18), let us give a little more heuristic details. Considering a Lebesgue function $f$, our bound (4.1) for the approximating sequence $\left(f_{j}\right)_{j \in \mathbf{N}} \subset L^{\infty}\left(E_{T}, \mathbf{R}_{\geq 0}\right)$ becomes $\left\|f_{j}\right\|_{L^{\gamma}\left(E_{T}\right)} \leq\|f\|_{L^{\gamma}\left(E_{T}\right)}<\infty$. Then, the quantity $\gamma$ is chosen optimal to the effect that after rewriting the integral (4.14) as

$$
\iint_{E_{T}} f_{j} \varphi d z \leq\left[\iint_{E_{T}}|f|^{\gamma} d z\right]^{\frac{1}{\gamma}}\left[\iint_{E_{T}}\left|u_{j}^{\alpha+1}\right|^{\frac{\gamma^{\prime}}{\alpha+1}} d z\right]^{\frac{1}{\gamma^{\prime}}}
$$


via Hölder's inequality, $\gamma$ is the smallest possible parameter such that the GagliardoNirenberg inequality is applicable to the last integral. By Young's inequality and a reabsorption argument, this eventually grants an energy estimate of the form claimed above. Note that, for $p=2$, the exponent $\gamma$ consorts with the upper bound for the number $s$ from [5, cond. (1.16)]. Furthermore, for $m=1, \gamma$ corresponds to the Hölder conjugate of $\frac{p(n+2)}{n}$ such that the integral $\iint_{E_{T}} f_{j} \varphi d z$ is well-defined for testing functions $\varphi \sim u_{j}$ due to the fact that, for $p$-Laplacian equations, we have $u_{j} \in$ $L^{\frac{p(n+2)}{n}}\left(E_{T}\right)$ by the Gagliardo-Nirenberg inequality. In this regard, the value of $\gamma$ is in perfect accordance with the earlier results for porous medium type and $p$-Laplacian equations. Under the additional premise that $\mu$ is not a Radon measure, but a Lebesgue function that is sufficiently regular in the described sense, the following reasoning can completely be performed with only minor modifications and yields the existence of a weak solution of the Cauchy-Dirichlet problem (1.2).

In the next lemma, we will establish a uniform estimate for $u_{j}^{\alpha}\left|D u_{j}^{\alpha+1}\right|^{p-1}$ in $L^{1}\left(E_{T}\right)$. For that purpose, we need to restrict the condition (4.7) for the parameter $q$.

Lemma 4.4. Under the assumptions of Lemma 4.2 with (4.7) being replaced by the prerequisite

$$
\max \left\{1, p-\frac{1}{\alpha+1}\right\} \leq q<p-\frac{n}{n(\alpha+1)+1}
$$

we have

$$
\iint_{E_{T}} u_{j}^{\alpha}\left|D u_{j}^{\alpha+1}\right|^{p-1} d z \leq c<\infty
$$

with a constant $c \equiv c\left(n, m, p, q, C_{0},\left|E_{T}\right|, \mu\left(E_{T}\right)\right)$. In particular, $c$ does not depend on $j$.

Proof. By Hölder's inequality, there holds

$$
\iint_{E_{T}} u_{j}^{\alpha}\left|D u_{j}^{\alpha+1}\right|^{p-1} d z \leq\left[\iint_{E_{T}} u_{j}^{\frac{\alpha q}{q-p+1}} d z\right]^{\frac{q-p+1}{q}}\left[\iint_{E_{T}}\left|D u_{j}^{\alpha+1}\right|^{q} d z\right]^{\frac{p-1}{q}} .
$$

Next, we recall the range (4.19) for $q$ and the uniform estimate in $L^{(\alpha+1) q}\left(E_{T}\right)$ for the sequence $\left(u_{j}\right)_{j \in \mathbf{N}}$ from (4.10). Hence, we obtain by another application of Hölder's inequality that the first integral is uniformly bounded. Then, (4.20) can be concluded with the help of the bound (4.9) for the second integral.

We remark that the constraint (4.19) for $q$ is in perfect accordance with the requirement $\frac{m-1}{m+1} \leq q-1$ from [5, Sec. 6.3.1] in the case $p=2$. Since the estimates (4.9) and (4.10) are uniform in $j$, they admit some weak limits $u$ of $u_{j}$ and $v$ of $D u_{j}^{\alpha+1}$, respectively. It has to be elucidated that the latter coincides with $D u^{\alpha+1}$, which we will accomplish in the next section.

4.3. Uniform bounds for the approximating sequence. We start with a fixed sequence $\left(u_{j}\right)_{j \in \mathbf{N}}$ of weak solutions of the Cauchy-Dirichlet problems (4.4), which exists by Lemma 4.1. By Lemma 4.2, we have

$$
\sup _{j \in \mathbf{N}}\left[\iint_{E_{T}}\left|u_{j}\right|^{(\alpha+1) q} d z+\iint_{E_{T}}\left|D u_{j}^{\alpha+1}\right|^{q} d z\right]<\infty
$$

for some $q>1$ as in (4.19), which shall be fixed for the rest of this paper. Therefore, there exist a subsequence (still denoted by $\left(u_{j}\right)_{j \in \mathbf{N}}$ ) and functions $u \in L^{(\alpha+1) q}\left(E_{T}\right)$ 
and $v \in L^{q}\left(E_{T}, \mathbf{R}^{n}\right)$ such that

$$
u_{j} \rightarrow u \text { weakly in } L^{(\alpha+1) q}\left(E_{T}\right) \text { as } j \rightarrow \infty
$$

and

$$
D u_{j}^{\alpha+1} \rightarrow v \text { weakly in } L^{q}\left(E_{T}, \mathbf{R}^{n}\right) \text { as } j \rightarrow \infty .
$$

Since, up to now, we only know the above weak convergences, the functions $u$ and $v$ might not be related. The main objective in what follows is the identification of the weak limit $v$ as $D u^{\alpha+1}$ using the strong convergence $u_{j} \rightarrow u$ in $L_{\text {loc }}^{s}\left(E_{T}\right)$ for some appropriate $s$ (see (4.31)).

4.3.1. Truncations from below and their a priori estimates. Let $\varepsilon>0$ be fixed and truncate the functions $u_{j}$ from below, i.e. consider the functions

$$
w_{j}^{(\varepsilon)}:=\max \left\{u_{j}, \varepsilon\right\}=\max \left\{u_{j}^{\alpha+1}, \varepsilon^{\alpha+1}\right\}^{\frac{1}{\alpha+1}} .
$$

We aim at applying [32, Cor. 4] to the set $\left\{w_{j}^{(\varepsilon)}: j \in \mathbf{N}\right\}$. Ergo, we have to ensure that $\left(w_{j}^{(\varepsilon)}\right)_{j \in \mathbf{N}}$ is bounded in $L^{q}\left((0, T) ; W^{1, q}(E)\right)$ and $\left(\partial_{t} w_{j}^{(\varepsilon)}\right)_{j \in \mathbf{N}}$ is bounded in $L_{\text {loc }}^{1}\left((0, T) ; W^{-1,1}(E)\right)$ (see (4.23) and (4.27), respectively). By the fact that $\left|w_{j}^{(\varepsilon)}\right| \leq u_{j}+\varepsilon$ on $E_{T}$ and the energy estimate (4.10), one can infer

$$
\iint_{E_{T}}\left|w_{j}^{(\varepsilon)}\right|^{q} d z \leq c \iint_{E_{T}} u_{j}^{q} d z+c \leq c<\infty
$$

with a constant $c \equiv c\left(n, m, p, q, C_{0},\left|E_{T}\right|, \mu\left(E_{T}\right), \varepsilon\right)$ independent of $j \in \mathbf{N}$. Next, we will establish a bound for $\left(D w_{j}^{(\varepsilon)}\right)_{j \in \mathbf{N}}$ in $L^{q}\left(E_{T}, \mathbf{R}^{n}\right)$. From Lemma 2.2, we deduce the pointwise bound

$$
\left|D w_{j}^{(\varepsilon)}\right|=\frac{1}{\alpha+1} \chi_{\left\{u_{j}>\varepsilon\right\}} u_{j}^{-\alpha}\left|D u_{j}^{\alpha+1}\right| \leq \frac{1}{\alpha+1} \varepsilon^{-\alpha}\left|D u_{j}^{\alpha+1}\right|
$$

on $E_{T}$ for the spatial derivative of $w_{j}^{(\varepsilon)}$. Thus, there holds

$$
\iint_{E_{T}}\left|D w_{j}^{(\varepsilon)}\right|^{q} d z \leq c \iint_{E_{T}}\left|D u_{j}^{\alpha+1}\right|^{q} d z \leq c<\infty
$$

with a constant $c \equiv c\left(n, m, p, q, C_{0},\left|E_{T}\right|, \mu\left(E_{T}\right), \varepsilon\right)$ independent of $j \in \mathbf{N}$, where we have used (4.9). Consequently, we arrive at

$$
\sup _{j \in \mathbf{N}}\left[\iint_{E_{T}}\left|w_{j}^{(\varepsilon)}\right|^{q} d z+\iint_{E_{T}}\left|D w_{j}^{(\varepsilon)}\right|^{q} d z\right]<\infty,
$$

i.e. $\left(w_{j}^{(\varepsilon)}\right)_{j \in \mathbf{N}}$ is bounded in $L^{q}\left((0, T) ; W^{1, q}(E)\right)$ for any $q$ as in (4.19).

4.3.2. Estimates for the time derivative. In the following, we will establish a bound in $L_{\text {loc }}^{1}\left((0, T) ; W^{-1,1}(E)\right)$ for $\left(\partial_{t} w_{j}^{(\varepsilon)}\right)_{j \in \mathbf{N}}$. For that purpose, we first note that $w_{j}^{(\varepsilon)}$ can be rewritten in the form

$$
w_{j}^{(\varepsilon)}=u_{j}+\varepsilon-\widehat{w}_{j}^{(\varepsilon)} \quad \text { with } \widehat{w}_{j}^{(\varepsilon)}:=\min \left\{u_{j}, \varepsilon\right\}
$$

such that we have to acquire uniform bounds in $L_{\text {loc }}^{1}\left((0, T) ; W^{-1,1}(E)\right)$ for the sequences $\left(\partial_{t} u_{j}\right)_{j \in \mathbf{N}}$ and $\left(\partial_{t} \widehat{w}_{j}^{(\varepsilon)}\right)_{j \in \mathbf{N}}$ (see (4.25) and (4.26), respectively). For $\varphi \in$ $C_{0}^{1}\left(E_{T}\right)$ with $\|\varphi\|_{L^{\infty}\left((0, T) ; W^{1, \infty}(E)\right)} \leq 1$, we start with the weak formulation (4.5) of 
the Cauchy-Dirichlet problem (4.4) and proceed by exerting the growth condition (1.4), the estimate (4.20), and the bound (4.1) to conclude that

$$
\left|\iint_{E_{T}} u_{j} \partial_{t} \varphi d z\right| \leq\left|\iint_{E_{T}} \mathbf{A}\left(x, t, u_{j}, D u_{j}\right) \cdot D \varphi d z\right|+\left|\iint_{E_{T}} \mu_{j} \varphi d z\right| \leq c<\infty
$$

with a constant $c \equiv c\left(n, m, p, q, C_{0}, C_{1},\left|E_{T}\right|, \mu\left(E_{T}\right)\right)$ independent of $j \in \mathbf{N}$. By taking the supremum over all $\varphi \in C_{0}^{1}\left(E_{T}\right)$ with $\|\varphi\|_{L^{\infty}\left((0, T) ; W^{1, \infty}(E)\right)} \leq 1$, we receive

$$
\left\|\partial_{t} u_{j}\right\|_{L^{1}\left((0, T) ; W^{-1,1}(E)\right)} \leq c<\infty \text {. }
$$

Hence, it remains to derive a similar (local) bound for $\partial_{t} \widehat{w}_{j}^{(\varepsilon)}$. By Lemma 3.3, we know that the functions $\widehat{w}_{j}^{(\varepsilon)}$ are bounded weak supersolutions of the doubly nonlinear parabolic equation (4.3), and therefore Lemma 3.8 is applicable. It yields the estimate

$$
\left\|\partial_{t} \widehat{w}_{j}^{(\varepsilon)}\right\|_{L^{1}\left(\left(t_{1}, t_{2}\right) ; W^{-1,1}(U)\right)} \leq c|W|\left[\frac{\varepsilon^{p+m-2}\left(t_{2}-t_{1}\right)}{\operatorname{dist}(U, \partial W)^{p}}+\varepsilon\right]
$$

for any $U \Subset W \Subset E$ with $\operatorname{dist}(U, \partial W) \leq 1,0<t_{1}<t_{2}<T$, and for any $j \in \mathbf{N}$, where the constant $c$ only depends on $m, p, C_{0}$ and $C_{1}$. Joining this with (4.25) and recalling (4.24), we obtain for any fixed $\varepsilon>0, U \Subset E$, and $0<t_{1}<t_{2}<T$ that

$$
\left\|\partial_{t} w_{j}^{(\varepsilon)}\right\|_{L^{1}\left(\left(t_{1}, t_{2}\right) ; W^{-1,1}(U)\right)} \leq c<\infty
$$

with a constant $c$ independent of $j \in \mathbf{N}$, i.e. we have found an $L^{1}\left(\left(t_{1}, t_{2}\right) ; W^{-1,1}(U)\right)$ bound for $\left(\partial_{t} w_{j}^{(\varepsilon)}\right)_{j \in \mathbf{N}}$.

4.3.3. Identifying the weak limit $\boldsymbol{v}$. In this step, we will identify with $D u^{\alpha+1}$ the limit $v$ of the weakly convergent sequence $\left(D u_{j}^{\alpha+1}\right)_{j \in \mathbf{N}}$. We will adapt the techniques from [27, p. $13 \mathrm{f}$.] and [5, Sec. 6.3.3] and slightly modify the reasoning whenever necessary. Take an exhaustion of $E$ by smooth sets $\left(U^{(\ell)}\right)_{\ell \in \mathbf{N}}$ and nested intervals $\left(\left(t_{1}^{(\ell)}, t_{2}^{(\ell)}\right)\right)_{\ell \in \mathbf{N}}$ with $U^{(\ell)} \Subset E$ and $0<t_{1}^{(\ell)}<t_{2}^{(\ell)}<T$ for any $\ell \in \mathbf{N}$, and such that

$$
E_{T}=\bigcup_{\ell=1}^{\infty}\left[U^{(\ell)} \times\left(t_{1}^{(\ell)}, t_{2}^{(\ell)}\right)\right]=: \bigcup_{\ell=1}^{\infty} \mathbf{U}^{(\ell)} .
$$

Now, for a fixed $\varepsilon>0$, we appeal to [32, Cor. 4] with respect to the set $\left\{w_{j}^{(\varepsilon)}: j \in \mathbf{N}\right\}$ and the following choice of spaces: $X:=W^{1,1}\left(U^{(1)}\right), B:=L^{1}\left(U^{(1)}\right)$, and $Y:=$ $W^{-1,1}\left(U^{(1)}\right)$. Note that this is possible due to the uniform bounds (4.23) and (4.27). We achieve the precompactness of the sequence $\left(w_{j}^{(\varepsilon)}\right)_{j \in \mathbf{N}}$ in $L^{1}\left(\mathbf{U}^{(1)}\right)$, which allows us to extract a subset $\mathfrak{K}_{1}^{(\varepsilon)} \subset \mathbf{N}$ such that

$$
w_{j}^{(\varepsilon)} \rightarrow \widetilde{w}_{1}^{(\varepsilon)} \text { in } L^{1}\left(\mathbf{U}^{(1)}\right) \text { and a.e. on } \mathbf{U}^{(1)} \text { as } \mathfrak{K}_{1}^{(\varepsilon)} \ni j \rightarrow \infty .
$$

On $\mathbf{U}^{(2)}$, we consider the sequence $\left(w_{j}^{(\varepsilon)}\right)_{j \in \mathfrak{K}_{1}^{(\varepsilon)}}$. The previous argument applies again and gives a subset $\mathfrak{K}_{2}^{(\varepsilon)} \subset \mathfrak{K}_{1}^{(\varepsilon)}$ such that

$$
w_{j}^{(\varepsilon)} \rightarrow \widetilde{w}_{2}^{(\varepsilon)} \text { in } L^{1}\left(\mathbf{U}^{(2)}\right) \text { and a.e. on } \mathbf{U}^{(2)} \text { as } \mathfrak{K}_{2}^{(\varepsilon)} \ni j \rightarrow \infty \text {. }
$$

Of course, there holds $\widetilde{w}_{2}^{(\varepsilon)} \equiv \widetilde{w}_{1}^{(\varepsilon)}$ on $\mathbf{U}^{(1)}$ since pointwise limits are unique. This process can be continued inductively by picking a subset $\mathfrak{K}_{\ell+1}^{(\varepsilon)} \subset \mathfrak{K}_{\ell}^{(\varepsilon)}$ such that the subsequence $\left(w_{j}^{(\varepsilon)}\right)_{j \in \mathfrak{K}_{\ell+1}^{(\varepsilon)}}$ converges strongly in $L^{1}\left(\mathbf{U}^{(\ell+1)}\right)$ and almost everywhere on 
$\mathbf{U}^{(\ell+1)}$ to some function $\widetilde{w}_{\ell+1}^{(\varepsilon)}$ as $\mathfrak{K}_{\ell+1}^{(\varepsilon)} \ni j \rightarrow \infty$. Clearly, we have $\widetilde{w}_{\ell+1}^{(\varepsilon)} \equiv \widetilde{w}_{\ell}^{(\varepsilon)}$ on $\mathbf{U}^{(\ell)}$. Now, let

$$
\mathfrak{K}_{\infty}^{(\varepsilon)}:=\bigcap_{\ell \in \mathbf{N}} \mathfrak{K}_{\ell}^{(\varepsilon)}=\lim _{\ell \rightarrow \infty} \mathfrak{K}_{\ell}^{(\varepsilon)}
$$

be the diagonal set. Then, for the corresponding diagonal sequence of functions $\left(w_{j}^{(\varepsilon)}\right)_{j \in \mathfrak{K}_{\infty}^{(\varepsilon)}}$, we conclude that

$$
w_{j}^{(\varepsilon)} \rightarrow w^{(\varepsilon)} \text { in } L_{\text {loc }}^{1}\left(E_{T}\right) \text { and a.e. on } E_{T} \text { as } \mathfrak{K}_{\infty}^{(\varepsilon)} \ni j \rightarrow \infty,
$$

where $w^{(\varepsilon)}$ is defined in a natural way by the local limits $\widetilde{w}_{\ell}^{(\varepsilon)}$, i.e. $w^{(\varepsilon)} \equiv \widetilde{w}_{\ell}^{(\varepsilon)}$ on $\mathbf{U}^{(\ell)}$. Next, we choose the values of $\varepsilon$ as $\varepsilon_{i}:=\frac{1}{i}(i \in \mathbf{N})$. As a result, the method from above shows that there exists a subset $\mathfrak{L}_{1}:=\mathfrak{K}_{\infty}^{\left(\varepsilon_{1}\right)} \subset \mathbf{N}$ such that

$$
w_{j}^{\left(\varepsilon_{1}\right)} \rightarrow w^{\left(\varepsilon_{1}\right)} \text { in } L_{\mathrm{loc}}^{1}\left(E_{T}\right) \text { and a.e. on } E_{T} \text { as } \mathfrak{L}_{1} \ni j \rightarrow \infty \text {. }
$$

Starting with $\left(w_{j}^{\left(\varepsilon_{2}\right)}\right)_{j \in \mathfrak{L}_{1}}$, the procedure yields another subset $\mathfrak{L}_{2} \subset \mathfrak{L}_{1}$ such that

$$
w_{j}^{\left(\varepsilon_{2}\right)} \rightarrow w^{\left(\varepsilon_{2}\right)} \text { in } L_{\text {loc }}^{1}\left(E_{T}\right) \text { and a.e. on } E_{T} \text { as } \mathfrak{L}_{2} \ni j \rightarrow \infty \text {. }
$$

Inductively, we find $\mathfrak{L}_{i+1} \subset \mathfrak{L}_{i}$ admitting the convergence

$$
w_{j}^{\left(\varepsilon_{i+1}\right)} \rightarrow w^{\left(\varepsilon_{i+1}\right)} \text { in } L_{\text {loc }}^{1}\left(E_{T}\right) \text { and a.e. on } E_{T} \text { as } \mathfrak{L}_{i+1} \ni j \rightarrow \infty \text {. }
$$

At this stage, we select again the diagonal set

$$
\mathfrak{L}_{\infty}:=\bigcap_{i \in \mathbf{N}} \mathfrak{L}_{i}=\lim _{i \rightarrow \infty} \mathfrak{L}_{i},
$$

and infer that, for any $i \in \mathbf{N}$, there holds

$$
w_{j}^{\left(\varepsilon_{i}\right)} \rightarrow w^{\left(\varepsilon_{i}\right)} \text { in } L_{\text {loc }}^{1}\left(E_{T}\right) \text { and a.e. on } E_{T} \text { as } \mathfrak{L}_{\infty} \ni j \rightarrow \infty \text {. }
$$

Since

$$
w_{j}^{\left(\varepsilon_{i}\right)}=\max \left\{u_{j}, \frac{1}{i}\right\} \geq \max \left\{u_{j}, \frac{1}{i+1}\right\}=w_{j}^{\left(\varepsilon_{i+1}\right)},
$$

the a.e.-convergence in (4.28) implies that

$$
w^{\left(\varepsilon_{i}\right)}(x, t)=\lim _{\mathfrak{L}_{\infty} \ni j \rightarrow \infty} w_{j}^{\left(\varepsilon_{i}\right)}(x, t) \geq \lim _{\mathfrak{L} \infty \ni j \rightarrow \infty} w_{j}^{\left(\varepsilon_{i+1}\right)}(x, t)=w^{\left(\varepsilon_{i+1}\right)}(x, t)
$$

for almost every $(x, t) \in E_{T}$, i.e. the sequence $\left(w^{\left(\varepsilon_{i}\right)}\right)_{i \in \mathbf{N}}$ of non-negative functions is nonincreasing and, therefore, concedes a pointwise, non-negative limit $w \in L_{\mathrm{loc}}^{1}\left(E_{T}\right)$. Thus,

$$
w(x, t):=\lim _{i \rightarrow \infty} w^{\left(\varepsilon_{i}\right)}(x, t)
$$

is well-defined for almost every $(x, t) \in E_{T}$. In view of the $L_{\text {loc }}^{1}\left(E_{T}\right)$-convergence of $\left(w_{j}^{\left(\varepsilon_{i}\right)}\right)_{j \in \mathfrak{L}_{\infty}}$ to $w^{\left(\varepsilon_{i}\right)}$ from (4.28), we have

$$
\iint_{U_{t_{1}, t_{2}}}\left|w^{\left(\varepsilon_{i}\right)}\right| d z=\lim _{\mathfrak{L}_{\infty} \ni j \rightarrow \infty} \iint_{U_{t_{1}, t_{2}}} w_{j}^{\left(\varepsilon_{i}\right)} d z \leq \sup _{j \in \mathbf{N}} \iint_{E_{T}} u_{j} d z+\left|E_{T}\right|<\infty
$$

for any $U \Subset E$ and $0<t_{1}<t_{2}<T$, where we also have used the definition of $w_{j}^{\left(\varepsilon_{i}\right)}$ and the energy estimate (4.8). Hence, the sequence of functions $\left(w^{\left(\varepsilon_{i}\right)}\right)_{i \in \mathbf{N}}$ is uniformly bounded in $L^{1}\left(U_{t_{1}, t_{2}}\right)$, and we conclude that

$$
w^{\left(\varepsilon_{i}\right)} \rightarrow w \text { in } L_{\mathrm{loc}}^{1}\left(E_{T}\right) \text { as } i \rightarrow \infty
$$


by the dominated convergence theorem. Next, we consider the difference between $w$ and $u_{j}$ in the $L_{\text {loc }}^{1}\left(E_{T}\right)$-norm and obtain for any $U \Subset E$ and $0<t_{1}<t_{2}<T$ that

$$
\begin{aligned}
\iint_{U_{t_{1}, t_{2}}}\left|w-u_{j}\right| d z \leq & \iint_{U_{t_{1}, t_{2}}}\left|w-w^{\left(\varepsilon_{i}\right)}\right| d z+\iint_{U_{t_{1}, t_{2}}}\left|w^{\left(\varepsilon_{i}\right)}-w_{j}^{\left(\varepsilon_{i}\right)}\right| d z \\
& +\iint_{U_{t_{1}, t_{2}}}\left|w_{j}^{\left(\varepsilon_{i}\right)}-u_{j}\right| d z .
\end{aligned}
$$

The third term appearing on the right-hand side of the preceding inequality can be estimated as follows:

$$
\iint_{U_{t_{1}, t_{2}}}\left|w_{j}^{\left(\varepsilon_{i}\right)}-u_{j}\right| d z=\iint_{U_{t_{1}, t_{2}}} \chi_{\left\{u_{j}<\varepsilon_{i}\right\}}\left|\frac{1}{i}-u_{j}\right| d z \leq \frac{1}{i}\left|E_{T}\right| .
$$

We plug this inequality in (4.30) and subsequently pass to the limit $\mathfrak{L}_{\infty} \ni j \rightarrow \infty$, taking into account that the second integral on the right-hand side of (4.30) converges to 0 by (4.28). Consequently, we arrive at

$$
\limsup _{\mathfrak{L}_{\infty} \ni j \rightarrow \infty} \iint_{U_{t_{1}, t_{2}}}\left|w-u_{j}\right| d z \leq \iint_{U_{t_{1}, t_{2}}}\left|w-w^{\left(\varepsilon_{i}\right)}\right| d z+\frac{1}{i}\left|E_{T}\right| .
$$

Letting $i \rightarrow \infty$ and relying on (4.29), we find that

$$
u_{j} \rightarrow w \text { in } L_{\text {loc }}^{1}\left(E_{T}\right) \text { and a.e. on } E_{T} \text { as } \mathfrak{L}_{\infty} \ni j \rightarrow \infty,
$$

and, by (4.21) and the uniqueness of weak limits, we receive $w \equiv u$ on $E_{T}$. Therefore, since, by (4.10) and (4.21), $u_{j}$ and $u$ are uniformly bounded in $L^{(\alpha+1) q}\left(E_{T}\right)$, we can exploit Lyapunov's inequality to conclude for any $1 \leq s<(\alpha+1) q$ that

$$
u_{j} \rightarrow u \text { in } L_{\text {loc }}^{s}\left(E_{T}\right) \text { and a.e. on } E_{T} \text { as } \mathfrak{L}_{\infty} \ni j \rightarrow \infty \text {. }
$$

We are now ready to identify the weak limit $v$ by the following computation. For any $\varphi \in C_{0}^{\infty}\left(E_{T}, \mathbf{R}^{n}\right)$, there holds

$$
\begin{aligned}
\iint_{E_{T}} v \cdot \varphi d z & =\lim _{\mathfrak{L}_{\infty} \ni j \rightarrow \infty} \iint_{E_{T}} D u_{j}^{\alpha+1} \cdot \varphi d z=-\lim _{\mathfrak{L}_{\infty} \ni j \rightarrow \infty} \iint_{E_{T}} u_{j}^{\alpha+1} \operatorname{div}(\varphi) d z \\
& =-\iint_{E_{T}} u^{\alpha+1} \operatorname{div}(\varphi) d z
\end{aligned}
$$

that is $v=D u^{\alpha+1}$ in the weak sense. Here, we have used in turn (4.22), an integration by parts as well as the convergence in (4.31). Thus, the identification of $v$ as $D u^{\alpha+1}$ is complete, and the convergence in (4.22) can be rewritten in the form

$$
D u_{j}^{\alpha+1} \rightarrow D u^{\alpha+1} \text { weakly in } L^{q}\left(E_{T}, \mathbf{R}^{n}\right) \text { as } \mathfrak{L}_{\infty} \ni j \rightarrow \infty .
$$

We terminate this paragraph remarking that, from now on, we assume that not only the subsequences $\left(u_{j}\right)_{j \in \mathfrak{L}_{\infty}}$ and $\left(D u_{j}^{\alpha+1}\right)_{j \in \mathfrak{L}_{\infty}}$, but the sequences $\left(u_{j}\right)_{j \in \mathbf{N}}$ and $\left(D u_{j}^{\alpha+1}\right)_{j \in \mathbf{N}}$ themselves, converge to $u$ and $D u^{\alpha+1}$, respectively, in order to avoid an overburdened notation.

4.3.4. Estimates for $\boldsymbol{u}$. Next, we examine how the bounds (4.8), (4.9) and (4.10) can be transferred from the approximating sequence to the limit function. One is able to deduce the following estimates for $u$.

Lemma 4.5. The limit function $u$ from (4.21) satisfies

$$
\sup _{t \in(0, T)} \int_{E \times\{t\}} u d x+\iint_{E_{T}}\left|D u^{\alpha+1}\right|^{q} d z+\iint_{E_{T}} u^{(\alpha+1) q} d z \leq c<\infty
$$


with a constant $c \equiv c\left(n, m, p, q, C_{0},\left|E_{T}\right|, \mu\left(E_{T}\right)\right)$, where $q$ is given by (4.19).

Proof. The claim follows directly from (4.8), (4.9), (4.10), (4.21), (4.32), and the weak lower semicontinuity of the $L^{\infty}\left((0, T) ; L^{1}(E)\right)-, L^{q}\left(E_{T}, \mathbf{R}^{n}\right)-$, and $L^{(\alpha+1) q}\left(E_{T}\right)$ norms.

It remains to show that the limit function $u$ is indeed a very weak solution of the Cauchy-Dirichlet problem (1.2), i.e. we have to prove that (1.10) holds true for any testing function $\varphi \in C^{1}\left(\overline{E_{T}}\right)$ vanishing on $[\partial E \times(0, T)] \cup[\bar{E} \times\{T\}]$. The crucial step in the proof will be the detection of the pointwise convergence of $D u_{j}^{\alpha+1}$ almost everywhere on $E_{T}$, which will be accomplished in the next paragraph. After that, the passage to the limit in the equation can be realized in Section 4.5. The methods are similar to the porous medium equation case (i.e. $p=2$ ), and our reasoning generalizes the arguments from [5, Sec. 6.4].

4.4. Pointwise convergence of $\boldsymbol{D} \boldsymbol{u}_{j}^{\alpha+1}$. In this section, we pursue the objective of extracting a subsequence of $\left(D u_{j}^{\alpha+1}\right)_{j \in \mathbf{N}}$ which admits the pointwise convergence to $D u^{\alpha+1}$ almost everywhere on $E_{T}$. For that purpose, let $\eta \in C_{0}^{\infty}(E,[0,1])$ be a cut-off function and define $u^{(\varepsilon)}:=\max \{u, \varepsilon\}$ for $\varepsilon>0$. We note that, by Lemma 2.2, the symbol $D u^{(\varepsilon)}$ for the weak spatial derivative of $u^{(\varepsilon)}$ has then to be understood in the sense of

$$
D u^{(\varepsilon)}=\frac{1}{\alpha+1} \chi_{E_{T} \cap\{u>\varepsilon\}} u^{-\alpha} D u^{\alpha+1} .
$$

Let $\ell>\varepsilon$ (later on, we will pass to the limits $\ell \rightarrow \infty$ and $\varepsilon \searrow 0$ such that this constraint is always satisfied) and define for $j \in \mathbf{N}$ the function

$$
\mathbf{h}_{j}^{(\varepsilon)}:=\frac{\left|\frac{1}{\alpha+1} D u_{j}^{\alpha+1}-u_{j}^{\alpha} D u^{(\varepsilon)}\right|^{\lambda}}{\left[\left|\frac{1}{\alpha+1} D u_{j}^{\alpha+1}-u_{j}^{\alpha} \mathbf{b}\left(\cdot, u_{j}\right)\right|^{p}+\left|u_{j}^{\alpha} D u^{(\varepsilon)}-u_{j}^{\alpha} \mathbf{b}\left(\cdot, u_{j}\right)\right|^{p}\right]^{\frac{\lambda-p}{p}}}
$$

on $E_{T}$, where $\lambda$ is the parameter from (1.6). In what follows, we consider the integral

$$
\begin{aligned}
& \iint_{E_{T}}\left[C_{2} \eta \mathbf{h}_{j}^{(\varepsilon)}\right]^{\vartheta} d z \\
& \leq \iint_{E_{T}}\left[\eta\left[\mathbf{A}\left(x, t, u_{j}, D u_{j}\right)-\mathbf{A}\left(x, t, u_{j}, D u^{(\varepsilon)}\right)\right] \cdot\left(D u_{j}-D u^{(\varepsilon)}\right)\right]^{\vartheta} d z \\
& =\iint_{E_{T} \cap\{u<\ell\}}[\cdots]^{\vartheta} d z+\iint_{E_{T} \cap\{u \geq \ell\}}[\cdots]^{\vartheta} d z=: \mathbf{J}_{j, \ell}(\varepsilon)+\mathbf{K}_{j, \ell}
\end{aligned}
$$

for some

$$
0<\vartheta<\frac{q(p+m-1)}{p(p+2 m-2)}<1,
$$

where $q$ is as in (4.19). In (4.36), we inserted the monotonicity assumption (1.6) and, in the second step, partitioned the domain of integration into the sets $E_{T} \cap\{u<\ell\}$ and $E_{T} \cap\{u \geq \ell\}$ (note that $\mathbf{K}_{j, \ell}$ is independent of the parameter $\varepsilon$ since $\ell>\varepsilon$ ). Before dealing with the term $\mathbf{J}_{j, \ell}(\varepsilon)$, we will analyze $\mathbf{K}_{j, \ell}$ in the next subsection.

4.4.1. The term $\mathbf{K}_{j, \ell}$. The above splitting with respect to level sets depending on large values of $\ell$ allows us to employ in the study of $\mathbf{K}_{j, \ell}$ that

$$
\left|E_{T} \cap\{u \geq \ell\}\right|=\iint_{E_{T} \cap\{u \geq \ell\}} 1 d z \leq \frac{1}{\ell} \iint_{E_{T}} u d z \rightarrow 0
$$


as $\ell \rightarrow \infty$, which holds true since $u \in L^{1}\left(E_{T}\right)$. Therefore, we will see in (4.40) that the term $\mathbf{K}_{j, \ell}$ converges to 0 in the limit $\ell \rightarrow \infty$ uniformly with respect to $j \in \mathbf{N}$. Using the fact that $0 \leq \eta \leq 1$, the growth condition (1.4), several standard estimates, the identity (4.34) and $\varepsilon<\ell$ (such that $\{u \geq \ell\} \subset\{u>\varepsilon\}$ ), and finally the lower bound $u \geq \ell$ on the domain of integration, we find that

$$
\begin{aligned}
\mathbf{K}_{j, \ell} \leq & \iint_{E_{T} \cap\{u \geq \ell\}}\left[\left(\left|\mathbf{A}\left(x, t, u_{j}, D u_{j}\right)\right|+\left|\mathbf{A}\left(x, t, u_{j}, D u^{(\varepsilon)}\right)\right|\right)\right. \\
& \left.\cdot\left(\left|D u_{j}\right|+\left|D u^{(\varepsilon)}\right|\right)\right]^{\vartheta} d z \\
\leq & c \iint_{E_{T} \cap\{u \geq \ell\}}\left[u_{j}^{m-1}\left(\left|D u_{j}\right|^{p}+\left|D u^{(\varepsilon)}\right|^{p}\right)\right]^{\vartheta} d z \\
\leq & c \iint_{E_{T} \cap\{u \geq \ell\}}\left[\left|D u_{j}^{\alpha+1}\right|^{p}+u_{j}^{m-1} u^{1-m}\left|D u^{\alpha+1}\right|^{p}\right]^{\vartheta} d z \\
\leq & c \iint_{E_{T} \cap\{u \geq \ell\}}\left|D u_{j}^{\alpha+1}\right|^{\vartheta p} d z+c \iint_{E_{T} \cap\{u \geq \ell\}}\left[\ell^{1-m} u_{j}^{m-1}\left|D u^{\alpha+1}\right|^{p}\right]^{\vartheta} d z \\
= & : \mathbf{K}^{(\mathbf{1})}+\mathbf{K}^{(2)}
\end{aligned}
$$

with a constant $c \equiv c\left(m, p, \vartheta, C_{1}\right)$. By Hölder's inequality and the energy estimate (4.9), the first term on the right-hand side simplifies to

$$
\mathbf{K}^{(1)} \leq c\left[\iint_{E_{T}}\left|D u_{j}^{\alpha+1}\right|^{q} d z\right]^{\frac{\vartheta p}{q}}\left|E_{T} \cap\{u \geq \ell\}\right|^{1-\frac{\vartheta p}{q}} \leq c\left|E_{T} \cap\{u \geq \ell\}\right|^{1-\frac{\vartheta p}{q}}
$$

with a constant $c \equiv c\left(n, m, p, q, \vartheta, C_{0}, C_{1},\left|E_{T}\right|, \mu\left(E_{T}\right)\right)$. Note that the last exponent is positive due to (4.37). For the second integral from (4.39), we apply Hölder's inequality twice and the estimates (4.33) and (4.10) to infer that

$$
\begin{aligned}
\mathbf{K}^{(2)} & \leq c \ell^{-\vartheta(m-1)}\left[\iint_{E_{T}}\left|D u^{\alpha+1}\right|^{q} d z\right]^{\frac{\vartheta p}{q}}\left[\iint_{E_{T} \cap\{u \geq \ell\}} u_{j}^{\frac{\vartheta(m-1) q}{q-\vartheta p}} d z\right]^{1-\frac{\vartheta p}{q}} \\
& \leq c \ell^{-\vartheta(m-1)}\left[\iint_{E_{T}} u_{j}^{(\alpha+1) q} d z\right]^{\frac{\vartheta(m-1)}{(\alpha+1) q}}\left|E_{T} \cap\{u \geq \ell\}\right|^{1-\frac{\vartheta(m-1)}{(\alpha+1)(q-\vartheta p)}} \\
& \leq c \ell^{-\vartheta(m-1)}\left|E_{T} \cap\{u \geq \ell\}\right|^{1-\frac{\vartheta(m-1)}{(\alpha+1)(q-\vartheta p)}}
\end{aligned}
$$

with a constant $c \equiv c\left(n, m, p, q, \vartheta, C_{0}, C_{1},\left|E_{T}\right|, \mu\left(E_{T}\right)\right)$, where the exponent $1-$ $\frac{\vartheta(m-1)}{(\alpha+1)(q-\vartheta p)}$ is also positive because of (4.37). Joining the preceding estimates with (4.39) and taking into account that (4.38) holds, we arrive at

$$
\lim _{\ell \rightarrow \infty} \sup _{j \in \mathbf{N}} \mathbf{K}_{j, \ell}=0,
$$

independent of $\varepsilon$. This finishes our investigation of $\mathbf{K}_{j, \ell}$, and we now turn our attention to the term $\mathbf{J}_{j, \ell}(\varepsilon)$ from (4.36). To this end, we first have to establish some properties of truncations.

4.4.2. Properties of truncations. For $\ell \in \mathbf{N}$, we define the truncation operators $T_{\ell}: \mathbf{R} \rightarrow \mathbf{R}$ by $T_{\ell}(s):=\max \{-\ell, \min \{s, \ell\}\}$. They satisfy $T_{\ell}(s)=\min \{s, \ell\}$ for $s \geq 0$. 
Lemma 4.6. Let $u_{j}$ be the weak solution from Lemma 4.1 and $u$ the limit function from (4.21). Then, there holds

$$
\iint_{E_{T}}\left|D\left[T_{\ell}\left(u_{j}\right)\right]^{\alpha+1}\right|^{p} d z \leq c \ell
$$

for any $\ell \in \mathbf{N}$ with a constant $c \equiv c\left(m, p, C_{0}, \mu\left(E_{T}\right)\right)$ independent of $j \in \mathbf{N}$. Furthermore, $T_{\ell}(u)^{\alpha+1}$ is weakly differentiable, and the derivative is given by the weak limit of $D\left[T_{\ell}\left(u_{j}\right)\right]^{\alpha+1}$, i.e. we have that

$$
D\left[T_{\ell}\left(u_{j}\right)\right]^{\alpha+1} \rightarrow D\left[T_{\ell}(u)\right]^{\alpha+1}
$$

converges weakly in $L^{p}\left(E_{T}, \mathbf{R}^{n}\right)$ as $j \rightarrow \infty$.

Proof. In (4.5), we employ the testing function $\varphi:=\zeta T_{\ell}\left(u_{j}\right)$, where $\zeta$ is the cutoff function from (4.11). As to the usage of time derivatives, we argue only formally again. For the first term appearing in the weak formulation, an integration by parts yields

$$
\begin{aligned}
\iint_{E_{T}} \zeta T_{\ell}\left(u_{j}\right) \partial_{t} u_{j} d z & =\iint_{E_{T}} \zeta T_{\ell}\left(u_{j}\right) \partial_{t} T_{\ell}\left(u_{j}\right) d z+\iint_{E_{T}} \zeta T_{\ell}\left(u_{j}\right) \partial_{t}\left[u_{j}-T_{\ell}\left(u_{j}\right)\right] d z \\
& =\frac{1}{2} \iint_{E_{T}} \zeta \partial_{t}\left|T_{\ell}\left(u_{j}\right)\right|^{2} d z+\ell \iint_{E_{T}} \zeta \partial_{t}\left(u_{j}-\ell\right)_{+} d z \\
& =\frac{1}{\delta} \iint_{E \times(\tau-\delta, \tau)}\left[\frac{1}{2}\left|T_{\ell}\left(u_{j}\right)\right|^{2}+\ell\left(u_{j}-\ell\right)_{+}\right] d z \geq 0,
\end{aligned}
$$

where $\tau \in(0, T)$ and $\delta \in(0, \tau)$. By treating the remaining two terms from (4.5) similarly to the computations performed in (4.13) and (4.14), one can show that (4.41) is valid. Therefore, $D\left[T_{\ell}\left(u_{j}\right)\right]^{\alpha+1}$ subconverges to some function $\widetilde{v} \in L^{p}\left(E_{T}, \mathbf{R}^{n}\right)$ weakly in $L^{p}\left(E_{T}, \mathbf{R}^{n}\right)$ as $j \rightarrow \infty$. Moreover, from (4.31), we know that $T_{\ell}\left(u_{j}\right)^{\alpha+1}$ converges to $T_{\ell}(u)^{\alpha+1}$ almost everywhere on $E_{T}$ as $j \rightarrow \infty$ such that, by the dominated convergence theorem, there holds

$$
\begin{aligned}
\iint_{E_{T}} \widetilde{v} \cdot \varphi d z & =\lim _{j \rightarrow \infty} \iint_{E_{T}} D\left[T_{\ell}\left(u_{j}\right)\right]^{\alpha+1} \cdot \varphi d z=-\lim _{j \rightarrow \infty} \iint_{E_{T}} T_{\ell}\left(u_{j}\right)^{\alpha+1} \operatorname{div}(\varphi) d z \\
& =-\iint_{E_{T}} T_{\ell}(u)^{\alpha+1} \operatorname{div}(\varphi) d z
\end{aligned}
$$

for any $\varphi \in C_{0}^{\infty}\left(E_{T}, \mathbf{R}^{n}\right)$, i.e. Lemma 4.6 is proven.

We have now collected all the necessary properties of the truncations and can devote ourselves to the term $\mathbf{J}_{j, \ell}(\varepsilon)$ from (4.36), where we priorly shall remember that $D u_{j}$ is given by (4.6).

4.4.3. The term $\mathbf{J}_{j, \ell}(\varepsilon)$. Let $\sigma>\varepsilon$ and $T_{\ell}$ as defined in Subsection 4.4.2. Availing ourselves of the identity $\chi_{\{u<\ell\}} D u^{(\varepsilon)}=\chi_{\left\{u^{(\varepsilon)}<\ell\right\}} D u^{(\varepsilon)}=D T_{\ell}\left(u^{(\varepsilon)}\right)$ and enlarging the domain of integration from $E_{T} \cap\{u<\ell\}$ to $E_{T}$, we can estimate the term $\mathbf{J}_{j, \ell}(\varepsilon)$ by

$$
\begin{aligned}
\mathbf{J}_{j, \ell}(\varepsilon) \leq & \iint_{E_{T}}\left[\eta\left[\mathbf{A}\left(x, t, u_{j}, D u_{j}\right)-\mathbf{A}\left(x, t, u_{j}, D T_{\ell}\left(u^{(\varepsilon)}\right)\right)\right]\right. \\
& \left.\cdot\left(D u_{j}-D T_{\ell}\left(u^{(\varepsilon)}\right)\right)\right]^{\vartheta} d z \\
= & : \mathbf{J}_{j, \ell}^{(+)}(\varepsilon, \sigma, h)+\mathbf{J}_{j, \ell}^{(-)}(\varepsilon, \sigma, h) .
\end{aligned}
$$


Here, in the second step, we have split the domain of integration $E_{T}$ into two parts $E_{T} \cap \Sigma_{j}^{+}$and $E_{T} \cap \Sigma_{j}^{-}$, where

$$
\Sigma_{j}^{+}:=\left\{\left|u_{j}-\llbracket T_{\ell}\left(u^{(\varepsilon)}\right) \rrbracket_{h}\right|>\sigma\right\} \quad \text { and } \quad \Sigma_{j}^{-}:=\left\{\left|u_{j}-\llbracket T_{\ell}\left(u^{(\varepsilon)}\right) \rrbracket_{h}\right| \leq \sigma\right\},
$$

and denoted the corresponding integrals by $\mathbf{J}_{j, \ell}^{(+)}(\varepsilon, \sigma, h)$ and $\mathbf{J}_{j, \ell}^{(-)}(\varepsilon, \sigma, h)$. First, we examine the former. For that purpose, we fix

$$
\vartheta:=\frac{q(p+m-1)}{2 p(p+2 m-2)}
$$

and note that this choice is allowed in (4.37). Then, using the fact that $0 \leq \eta \leq 1$, the growth condition (1.4), several standard estimates, the bound $T_{\ell}\left(u^{(\varepsilon)}\right) \geq \varepsilon$, the formula from Lemma 2.2 for the spatial derivative of $T_{\ell}\left(u^{(\varepsilon)}\right)$, Hölder's inequality (multiple times) as well as the estimates (4.9), (4.10) and (4.33), we obtain

$$
\begin{aligned}
\mathbf{J}_{j, \ell}^{(+)}(\varepsilon, \sigma, h) \leq & \iint_{E_{T} \cap \Sigma_{j}^{+}}\left[\left(\left|\mathbf{A}\left(x, t, u_{j}, D u_{j}\right)\right|+\left|\mathbf{A}\left(x, t, u_{j}, D T_{\ell}\left(u^{(\varepsilon)}\right)\right)\right|\right)\right. \\
& \left.\cdot\left(\left|D u_{j}\right|+\left|D T_{\ell}\left(u^{(\varepsilon)}\right)\right|\right)\right]^{\vartheta} d z \\
\leq & c \iint_{E_{T} \cap \Sigma_{j}^{+}}\left[u_{j}^{m-1}\left(\left|D u_{j}\right|^{p}+\left|D T_{\ell}\left(u^{(\varepsilon)}\right)\right|^{p}\right)\right]^{\vartheta} d z \\
\leq & c \iint_{E_{T} \cap \Sigma_{j}^{+}}\left[\left|D u_{j}^{\alpha+1}\right|^{\vartheta p}+\left(\frac{u_{j}}{\varepsilon}\right)^{\vartheta(m-1)}\left|D\left[T_{\ell}\left(u^{(\varepsilon)}\right)\right]^{\alpha+1}\right|^{\vartheta p}\right] d z \\
\leq & c\left|E_{T} \cap \Sigma_{j}^{+}\right|^{\frac{1}{2}} \cdot\left[\iint_{E_{T}}\left|D u_{j}^{\alpha+1}\right|^{2 \vartheta p} d z\right. \\
& \left.+\iint_{E_{T}}\left(\frac{u_{j}}{\varepsilon}\right)^{2 \vartheta(m-1)}\left|D\left[T_{\ell}\left(u^{(\varepsilon)}\right)\right]^{\alpha+1}\right|^{2 \vartheta p} d z\right]^{\frac{1}{2}} \\
\leq & c\left|E_{T} \cap \Sigma_{j}^{+}\right|^{\frac{1}{2}} \\
& \cdot\left(c+c_{\varepsilon}\left[\iint_{E_{T}} u_{j}^{(\alpha+1) q} d z\right]^{\frac{m-1}{p+2 m-2}}\left[\iint_{E_{T}}\left|D u^{\alpha+1}\right|^{q} d z\right]^{\frac{p+m-1}{p+2 m-2}}\right)^{\frac{1}{2}} \\
\leq & c_{\varepsilon}\left|E_{T} \cap\left\{\left|u_{j}-\llbracket T_{\ell}\left(u^{(\varepsilon)}\right) \rrbracket_{h}\right|>\sigma\right\}\right|^{\frac{1}{2}}
\end{aligned}
$$

with a constant $c_{\varepsilon} \equiv c_{\varepsilon}\left(n, m, p, q, C_{0}, C_{1},\left|E_{T}\right|, \mu\left(E_{T}\right), \varepsilon\right)$. We can now pass to the limits $j \rightarrow \infty$ and $h \searrow 0$ to conclude that

$$
\begin{aligned}
\lim _{h \searrow 0} \limsup _{j \rightarrow \infty} \mathbf{J}_{j, \ell}^{(+)}(\varepsilon, \sigma, h) & \leq c_{\varepsilon}\left|E_{T} \cap\left\{\left|u-T_{\ell}\left(u^{(\varepsilon)}\right)\right|>\sigma\right\}\right|^{\frac{1}{2}} \\
& =c_{\varepsilon}\left|E_{T} \cap\left\{\left|u-T_{\ell}(u)\right|>\sigma\right\}\right|^{\frac{1}{2}},
\end{aligned}
$$

where, in the last line, we used the fact that $\varepsilon<\sigma$, which implies that $\left|u-T_{\ell}\left(u^{(\varepsilon)}\right)\right|<$ $\sigma$ on the set $\{u<\varepsilon\}$. Letting $\ell \rightarrow \infty$, we infer

$$
\limsup _{\ell \rightarrow \infty} \lim _{h \searrow 0} \limsup _{j \rightarrow \infty} \mathbf{J}_{j, \ell}^{(+)}(\varepsilon, \sigma, h)=0
$$


for any pair of parameters $0<\varepsilon<\sigma$. Next, we turn our attention to the term $\mathbf{J}_{j, \ell}^{(-)}(\varepsilon, \sigma, h)$ from (4.42). Here, Hölder's inequality leads to

$$
\begin{aligned}
\mathbf{J}_{j, \ell}^{(-)}(\varepsilon, \sigma, h) \leq & \left|E_{T}\right|^{1-\vartheta}\left[\iint_{E_{T}} \chi_{\Sigma_{j}^{-}} \eta \mathbf{A}\left(x, t, u_{j}, D u_{j}\right) \cdot\left(D u_{j}-D T_{\ell}\left(u^{(\varepsilon)}\right)\right) d z\right. \\
& \left.-\iint_{E_{T}} \chi_{\Sigma_{j}^{-}} \eta \mathbf{A}\left(x, t, u_{j}, D T_{\ell}\left(u^{(\varepsilon)}\right)\right) \cdot\left(D u_{j}-D T_{\ell}\left(u^{(\varepsilon)}\right)\right) d z\right]^{\vartheta} \\
= & :\left|E_{T}\right|^{1-\vartheta}\left[\mathbf{J}_{1}^{(-)}-\mathbf{J}_{\mathbf{2}}^{(-)}\right]^{\vartheta}
\end{aligned}
$$

for $\vartheta$ as in (4.43). In the study of $\mathbf{J}_{j, \ell}^{(-)}(\varepsilon, \sigma, h)$, we start with the term $\mathbf{J}_{\mathbf{2}}^{(-)}$, rewriting the integrand in the following way:

$$
\begin{aligned}
& \mathbf{A}\left(\cdot, u_{j}, D T_{\ell}\left(u^{(\varepsilon)}\right)\right) \cdot\left(D u_{j}-D T_{\ell}\left(u^{(\varepsilon)}\right)\right) \\
& =\chi_{\left\{u_{j}>0\right\}} u_{j}^{-\alpha} \mathbf{A}\left(\cdot, u_{j}, D T_{\ell}\left(u^{(\varepsilon)}\right)\right) \cdot\left[\frac{1}{\alpha+1} D u_{j}^{\alpha+1}-u_{j}^{\alpha} D T_{\ell}\left(u^{(\varepsilon)}\right)\right] .
\end{aligned}
$$

Now, if $z=(x, t) \in E_{T}$ is such that $u_{j}(z) \rightarrow u(z)>0$ as $j \rightarrow \infty$, there holds

$$
\chi_{\left\{u_{j}>0\right\}}(z) u_{j}(z)^{-\alpha} \mathbf{A}\left(x, t, u_{j}(z), D T_{\ell}\left(u^{(\varepsilon)}\right)(z)\right) \rightarrow u(z)^{-\alpha} \mathbf{A}\left(x, t, u(z), D T_{\ell}\left(u^{(\varepsilon)}\right)(z)\right)
$$

as $j \rightarrow \infty$, while if $u_{j}(z) \rightarrow 0$ as $j \rightarrow \infty$, then, by the growth condition (1.4), we have

$$
\begin{aligned}
& \left|\chi_{\left\{u_{j}>0\right\}}(z) u_{j}(z)^{-\alpha} \mathbf{A}\left(x, t, u_{j}(z), D T_{\ell}\left(u^{(\varepsilon)}\right)(z)\right)\right| \\
& \leq C_{1} u_{j}(z)^{\alpha(p-1)}\left|D T_{\ell}\left(u^{(\varepsilon)}\right)(z)\right|^{p-1} \rightarrow 0
\end{aligned}
$$

as $j \rightarrow \infty$, so that

$$
\chi_{\left\{u_{j}>0\right\}} u_{j}^{-\alpha} \mathbf{A}\left(\cdot, u_{j}, D T_{\ell}\left(u^{(\varepsilon)}\right)\right) \rightarrow \chi_{\{u>0\}} u^{-\alpha} \mathbf{A}\left(\cdot, u, D T_{\ell}\left(u^{(\varepsilon)}\right)\right)
$$

converges almost everywhere on $E_{T}$ as $j \rightarrow \infty$. Hence, by applying Egorov's theorem for a given $\delta>0$, we find a compact set $K_{\delta} \subset E_{T}$ such that $\left|E_{T} \backslash K_{\delta}\right|<\delta$ and

$$
\chi_{\left\{u_{j}>0\right\}} u_{j}^{-\alpha} \mathbf{A}\left(\cdot, u_{j}, D T_{\ell}\left(u^{(\varepsilon)}\right)\right) \rightarrow \chi_{\{u>0\}} u^{-\alpha} \mathbf{A}\left(\cdot, u, D T_{\ell}\left(u^{(\varepsilon)}\right)\right)
$$

converges uniformly on $K_{\delta}$ as $j \rightarrow \infty$. Besides, from (4.31) and the dominated convergence theorem, there follows

$$
\chi_{\Sigma_{j}^{-}}=\chi_{\left\{\left|u_{j}-\llbracket T_{\ell}(u(\varepsilon)) \rrbracket_{h}\right| \leq \sigma\right\}} \rightarrow \chi_{\left\{\left|u-\llbracket T_{\ell}(u(\varepsilon)) \rrbracket_{h}\right| \leq \sigma\right\}}=: \chi_{\Sigma^{-}}
$$

in $L^{\omega}\left(E_{T}\right)$ as $j \rightarrow \infty$ for any $\omega \geq 1$ and almost every $\sigma>\varepsilon$. Now, by the triangle inequality, we obtain

$$
\begin{aligned}
& \left|\iint_{K_{\delta}}\left[\chi_{\Sigma_{j}^{-}} \eta \mathbf{A}\left(x, t, u_{j}, D T_{\ell}\left(u^{(\varepsilon)}\right)\right) \cdot D u_{j}-\chi_{\Sigma^{-}} \eta \mathbf{A}\left(x, t, u, D T_{\ell}\left(u^{(\varepsilon)}\right)\right) \cdot D u\right] d z\right| \\
& \leq c\left\|\mathbf{A}\left(\cdot, u_{j}, D T_{\ell}\left(u^{(\varepsilon)}\right)\right) \chi_{\left\{u_{j}>0\right\}} u_{j}^{-\alpha}-\mathbf{A}\left(\cdot, u, D T_{\ell}\left(u^{(\varepsilon)}\right)\right) \chi_{\{u>0\}} u^{-\alpha}\right\|_{\infty}\left\|D u_{j}^{\alpha+1}\right\|_{q} \\
& \quad+c\left\|\mathbf{A}\left(\cdot, u, D T_{\ell}\left(u^{(\varepsilon)}\right)\right) \chi_{\{u>0\}} u^{-\alpha}\right\|_{\infty}\left\|D u_{j}^{\alpha+1}\right\|\left\|_{q}\right\| \chi_{\Sigma_{j}^{-}}-\chi_{\Sigma^{-}} \|_{q^{\prime}} \\
& \quad+c\left|\iint_{K_{\delta}}\left[\chi_{\Sigma^{-}} \eta \mathbf{A}\left(x, t, u, D T_{\ell}\left(u^{(\varepsilon)}\right)\right) \chi_{\{u>0\}} u^{-\alpha}\right] \cdot\left(D u_{j}^{\alpha+1}-D u^{\alpha+1}\right) d z\right|
\end{aligned}
$$


with a constant $c \equiv c\left(m, p,\left|E_{T}\right|\right)$. Therefore, due to (4.46), (4.9), (4.47) and (4.32), we infer

$$
\begin{aligned}
& \lim _{j \rightarrow \infty} \iint_{K_{\delta}} \chi_{\Sigma_{j}^{-}} \eta \mathbf{A}\left(x, t, u_{j}, D T_{\ell}\left(u^{(\varepsilon)}\right)\right) \cdot D u_{j} d z \\
& =\iint_{K_{\delta}} \chi_{\Sigma^{-}} \eta \mathbf{A}\left(x, t, u, D T_{\ell}\left(u^{(\varepsilon)}\right)\right) \cdot D u d z .
\end{aligned}
$$

Remember that the symbols $D u_{j}$ and $D u$ have to be interpreted as in (4.6) and (1.11), respectively. For the other summand of the integral $\mathbf{J}_{\mathbf{2}}^{(-)}$, we compute

$$
\begin{aligned}
& \lim _{j \rightarrow \infty} \iint_{K_{\delta}} \chi_{\Sigma_{j}^{-}} \eta \mathbf{A}\left(x, t, u_{j}, D T_{\ell}\left(u^{(\varepsilon)}\right)\right) \cdot D T_{\ell}\left(u^{(\varepsilon)}\right) d z \\
& =\iint_{K_{\delta}} \chi_{\Sigma^{-}} \eta \mathbf{A}\left(x, t, u, D T_{\ell}\left(u^{(\varepsilon)}\right)\right) \cdot D T_{\ell}\left(u^{(\varepsilon)}\right) d z .
\end{aligned}
$$

Here, we used that $\mathbf{A}\left(\cdot, u_{j}, D T_{\ell}\left(u^{(\varepsilon)}\right)\right) \rightarrow \mathbf{A}\left(\cdot, u, D T_{\ell}\left(u^{(\varepsilon)}\right)\right)$ converges almost everywhere on $E_{T}$ as $j \rightarrow \infty$ because of (4.31) and the continuity of $\mathbf{A}\left(x, t, \cdot, D T_{\ell}\left(u^{(\varepsilon)}\right)\right)$. Thus, taking into account that, by (4.47), we can extract a (nonrelabeled) a.e.convergent subsequence of $\left(\chi_{\Sigma_{j}^{-}}\right)_{j \in \mathbf{N}}$, we also get that $\chi_{\Sigma_{j}^{-}} \mathbf{A}\left(\cdot, u_{j}, D T_{\ell}\left(u^{(\varepsilon)}\right)\right)$ converges almost everywhere on $E_{T}$ to $\chi_{\Sigma^{-}} \mathbf{A}\left(\cdot, u, D T_{\ell}\left(u^{(\varepsilon)}\right)\right)$ as $j \rightarrow \infty$. Since Egorov's theorem again provides the uniform convergence on $K_{\delta}$, we conclude that (4.49) is valid. It remains to control the integral $\mathbf{J}_{2}^{(-)}$on the complement $E_{T} \backslash K_{\delta}$. For that purpose, we first estimate

$$
\begin{aligned}
& \left|\chi_{\Sigma_{j}^{-}} \eta \mathbf{A}\left(\cdot, u_{j}, D T_{\ell}\left(u^{(\varepsilon)}\right)\right) \cdot D u_{j}\right| \leq C_{1} \chi_{\Sigma_{j}^{-}} u_{j}^{m-1}\left|D T_{\ell}\left(u^{(\varepsilon)}\right)\right|^{p-1}\left|D u_{j}\right| \\
& =C_{1} \chi_{\Sigma_{j}^{-}} T_{\ell+\sigma}\left(u_{j}\right)^{m-1}\left|D T_{\ell}\left(u^{(\varepsilon)}\right)\right|^{p-1}\left|D T_{\ell+\sigma}\left(u_{j}\right)\right| \\
& \leq c T_{\ell+\sigma}\left(u_{j}\right)^{\alpha(p-1)} T_{\ell}\left(u^{(\varepsilon)}\right)^{-\alpha(p-1)}\left|D\left[T_{\ell}\left(u^{(\varepsilon)}\right)\right]^{\alpha+1}\right|^{p-1}\left|D\left[T_{\ell+\sigma}\left(u_{j}\right)\right]^{\alpha+1}\right| \\
& \leq c\left[\frac{\ell+\sigma}{\varepsilon}\right]^{\alpha(p-1)}\left|D\left[T_{\ell}\left(u^{(\varepsilon)}\right)\right]^{\alpha+1}\right|^{p-1}\left|D\left[T_{\ell+\sigma}\left(u_{j}\right)\right]^{\alpha+1}\right|
\end{aligned}
$$

with a constant $c \equiv c\left(m, p, C_{1}\right)$, where, in the second step, we have exerted the definition of $\Sigma_{j}^{-}$and the fact that $\llbracket T_{\ell}\left(u^{(\varepsilon)}\right) \rrbracket_{h} \in[0, \ell]$. If we integrate on $E_{T} \backslash K_{\delta}$ and apply Hölder's inequality and subsequently the estimate (4.41), then, since $D\left[T_{\ell}(u)\right]^{\alpha+1} \in L^{p}\left(E_{T}, \mathbf{R}^{n}\right)$ by Lemma 4.6 , we find by the absolute continuity of the Lebesgue integral that

$$
\begin{aligned}
& \left|\iint_{E_{T} \backslash K_{\delta}} \chi_{\Sigma_{j}^{-}} \eta \mathbf{A}\left(x, t, u_{j}, D T_{\ell}\left(u^{(\varepsilon)}\right)\right) \cdot D u_{j} d z\right| \\
& \leq c\left[\frac{\ell+\sigma}{\varepsilon}\right]^{\alpha(p-1)}\left[\iint_{E_{T} \backslash K_{\delta}}\left|D\left[T_{\ell}\left(u^{(\varepsilon)}\right)\right]^{\alpha+1}\right|^{p} d z\right]^{\frac{1}{p^{\prime}}} \\
& \quad \cdot\left[\iint_{E_{T}}\left|D\left[T_{\ell+\sigma}\left(u_{j}\right)\right]^{\alpha+1}\right|^{p} d z\right]^{\frac{1}{p}} \\
& \leq c\left[\frac{\ell+\sigma}{\varepsilon}\right]^{\alpha(p-1)}\left[\iint_{E_{T} \backslash K_{\delta}}\left|D\left[T_{\ell}(u)\right]^{\alpha+1}\right|^{p} d z\right]^{\frac{1}{p^{\prime}}}[\ell+\sigma]^{\frac{1}{p}} \rightarrow 0
\end{aligned}
$$

in the limit $\delta \searrow 0$ uniformly with respect to $j$, where the constant $c$ only depends on $n, m, p, C_{0}, C_{1},\left|E_{T}\right|$ and $\mu\left(E_{T}\right)$. As for the other term of $\mathbf{J}_{2}^{(-)}$, we analogously 
estimate

$$
\begin{aligned}
\left|\chi_{\Sigma_{j}^{-}} \eta \mathbf{A}\left(\cdot, u_{j}, D T_{\ell}\left(u^{(\varepsilon)}\right)\right) \cdot D T_{\ell}\left(u^{(\varepsilon)}\right)\right| & \leq C_{1} \chi_{\Sigma_{j}^{-}} u_{j}^{m-1}\left|D T_{\ell}\left(u^{(\varepsilon)}\right)\right|^{p} \\
& =C_{1} \chi_{\Sigma_{j}^{-}} T_{\ell+\sigma}\left(u_{j}\right)^{m-1}\left|D T_{\ell}\left(u^{(\varepsilon)}\right)\right|^{p} \\
& \leq c T_{\ell+\sigma}\left(u_{j}\right)^{m-1} T_{\ell}\left(u^{(\varepsilon)}\right)^{1-m}\left|D\left[T_{\ell}\left(u^{(\varepsilon)}\right)\right]^{\alpha+1}\right|^{p} \\
& \leq c\left[\frac{\ell+\sigma}{\varepsilon}\right]^{m-1}\left|D\left[T_{\ell}\left(u^{(\varepsilon)}\right)\right]^{\alpha+1}\right|^{p},
\end{aligned}
$$

hence, again employing the absolute continuity of the Lebesgue integral, we infer

$$
\begin{aligned}
& \left|\iint_{E_{T} \backslash K_{\delta}} \chi_{\Sigma_{j}^{-}} \eta \mathbf{A}\left(x, t, u_{j}, D T_{\ell}\left(u^{(\varepsilon)}\right)\right) \cdot D T_{\ell}\left(u^{(\varepsilon)}\right) d z\right| \\
& \leq c\left[\frac{\ell+\sigma}{\varepsilon}\right]^{m-1} \iint_{E_{T} \backslash K_{\delta}}\left|D\left[T_{\ell}(u)\right]^{\alpha+1}\right|^{p} d z \rightarrow 0
\end{aligned}
$$

in the limit $\delta \searrow 0$ uniformly with respect to $j$. Using (4.48)-(4.51) and recalling (1.11), we obtain for the term $\mathbf{J}_{2}^{(-)}$from (4.45):

$$
\begin{aligned}
\left|\lim _{j \rightarrow \infty} \mathbf{J}_{\mathbf{2}}^{(-)}\right| & =\left|\iint_{E_{T}} \chi_{\Sigma^{-}} \eta \mathbf{A}\left(x, t, u, D T_{\ell}\left(u^{(\varepsilon)}\right)\right) \cdot\left(D u-D T_{\ell}\left(u^{(\varepsilon)}\right)\right) d z\right| \\
& \leq C_{1} \iint_{E_{T} \cap[\{u<\varepsilon\} \cup\{u>\ell\}]} u^{m-1}\left|D T_{\ell}\left(u^{(\varepsilon)}\right)\right|^{p-1}|D u| d z=0 .
\end{aligned}
$$

Here, we exploited the facts that $D T_{\ell}\left(u^{(\varepsilon)}\right)=D u$ on $E_{T} \cap\{\varepsilon \leq u \leq \ell\}$ and $D T_{\ell}\left(u^{(\varepsilon)}\right)=0$ on $E_{T} \cap[\{u<\varepsilon\} \cup\{u>\ell\}]$ as well as the growth condition (1.4). We now need to treat the leftover integral $\mathbf{J}_{\mathbf{1}}^{(-)}$from (4.45). First, we compute

$$
\chi_{E_{T} \cap \Sigma_{j}^{-}}\left(u_{j}-\llbracket T_{\ell}\left(u^{(\varepsilon)}\right) \rrbracket_{h}\right)=\chi_{E_{T} \cap \Sigma_{j}^{-}} T_{\sigma}\left(u_{j}-\llbracket T_{\ell}\left(u^{(\varepsilon)}\right) \rrbracket_{h}\right),
$$

which is why we have

$$
D u_{j}-D T_{\ell}\left(u^{(\varepsilon)}\right)=D\left[T_{\sigma}\left(u_{j}-\llbracket T_{\ell}\left(u^{(\varepsilon)}\right) \rrbracket_{h}\right)\right]+D \llbracket T_{\ell}\left(u^{(\varepsilon)}\right) \rrbracket_{h}-D T_{\ell}\left(u^{(\varepsilon)}\right)
$$

on $E_{T} \cap \Sigma_{j}^{-}$. Since $D\left[T_{\sigma}\left(u_{j}-\llbracket T_{\ell}\left(u^{(\varepsilon)}\right) \rrbracket_{h}\right)\right]=0$ on $E_{T} \backslash \Sigma_{j}^{-}$, there holds

$$
\begin{aligned}
\mathbf{J}_{\mathbf{1}}^{(-)}= & \iint_{E_{T}} \chi_{\Sigma_{j}^{-}} \eta \mathbf{A}\left(x, t, u_{j}, D u_{j}\right) \cdot\left[D \llbracket T_{\ell}\left(u^{(\varepsilon)}\right) \rrbracket_{h}-D T_{\ell}\left(u^{(\varepsilon)}\right)\right] d z \\
& +\iint_{E_{T}} \eta \mathbf{A}\left(x, t, u_{j}, D u_{j}\right) \cdot D T_{\sigma}\left(u_{j}-\llbracket T_{\ell}\left(u^{(\varepsilon)}\right) \rrbracket_{h}\right) d z .
\end{aligned}
$$

We observe that the first integral disappears in the limit $h \searrow 0$. To deal with the second term, we rely on the weak formulation of (4.4) and choose $\varphi:=\eta T_{\sigma}\left(u_{j}-\right.$ $\llbracket T_{\ell}\left(u^{(\varepsilon)}\right) \rrbracket_{h}$ ) as a testing function (note that $\varphi=\eta=0$ on $\partial E \times(0, T)$ ). This yields

$$
\begin{aligned}
& \iint_{E_{T}} \eta \mathbf{A}\left(x, t, u_{j}, D u_{j}\right) \cdot D T_{\sigma}\left(u_{j}-\llbracket T_{\ell}\left(u^{(\varepsilon)}\right) \rrbracket_{h}\right) d z \\
& =\iint_{E_{T}} \eta \mu_{j} T_{\sigma}\left(u_{j}-\llbracket T_{\ell}\left(u^{(\varepsilon)}\right) \rrbracket_{h}\right) d z-\iint_{E_{T}} \eta \partial_{t} u_{j} T_{\sigma}\left(u_{j}-\llbracket T_{\ell}\left(u^{(\varepsilon)}\right) \rrbracket_{h}\right) d z \\
& \quad-\iint_{E_{T}} T_{\sigma}\left(u_{j}-\llbracket T_{\ell}\left(u^{(\varepsilon)}\right) \rrbracket_{h}\right) \mathbf{A}\left(x, t, u_{j}, D u_{j}\right) \cdot D \eta d z \\
& =: \mathbf{L}_{\mathbf{1}}+\mathbf{L}_{\mathbf{2}}+\mathbf{L}_{\mathbf{3}} .
\end{aligned}
$$


Remember that basically we would have to apply the time mollification as done in (2.4) since the time derivative $\partial_{t} u_{j}$ does not need to exist, the details, however, will be omitted as this is a standard procedure. For the first term, (4.1) leads us to

$$
\left|\mathbf{L}_{1}\right| \leq\|\eta\|_{L^{\infty}(E)} \sigma \iint_{E_{T}} \mu_{j} d z \leq c \sigma
$$

with a constant $c=\mu\left(E_{T}\right)$. Moreover, availing ourselves of the growth condition (1.4) and the estimate (4.20), we find

$$
\left|\mathbf{L}_{\mathbf{3}}\right| \leq c \sigma \iint_{E_{T}} u_{j}^{\alpha}\left|D u_{j}^{\alpha+1}\right|^{p-1} d z \leq c \sigma
$$

with a constant $c \equiv c\left(n, m, p, q, C_{0}, C_{1},\left|E_{T}\right|, \mu\left(E_{T}\right),\|D \eta\|_{L^{\infty}\left(E, \mathbf{R}^{n}\right)}\right)$. Eventually, we rewrite $\mathbf{L}_{2}$ as follows:

$$
\begin{aligned}
\mathbf{L}_{\mathbf{2}}= & -\iint_{E_{T}} \eta \partial_{t} \llbracket T_{\ell}\left(u^{(\varepsilon)}\right) \rrbracket_{h} T_{\sigma}\left(u_{j}-\llbracket T_{\ell}\left(u^{(\varepsilon)}\right) \rrbracket_{h}\right) d z \\
& -\iint_{E_{T}} \eta \partial_{t}\left[u_{j}-\llbracket T_{\ell}\left(u^{(\varepsilon)}\right) \rrbracket_{h}\right] T_{\sigma}\left(u_{j}-\llbracket T_{\ell}\left(u^{(\varepsilon)}\right) \rrbracket_{h}\right) d z \\
= & : \mathbf{L}_{\mathbf{2}}^{(\mathbf{1})}+\mathbf{L}_{\mathbf{2}}^{(\mathbf{2})} .
\end{aligned}
$$

For the second integral, defining $\phi_{\sigma}(s):=\int_{0}^{s} T_{\sigma}(\tau) d \tau$ for $s \in \mathbf{R}$, and recalling that $u_{j}(\cdot, 0)=0=\llbracket T_{\ell}\left(u^{(\varepsilon)}\right) \rrbracket_{h}(\cdot, 0)$ on $E$, we obtain

$$
\begin{aligned}
\mathbf{L}_{\mathbf{2}}^{(\mathbf{2})} & =-\int_{E} \int_{0}^{T} \eta \partial_{t} \phi_{\sigma}\left(\left[u_{j}-\llbracket T_{\ell}\left(u^{(\varepsilon)}\right) \rrbracket_{h}\right](\cdot, t)\right) d t d x \\
& =\left[-\int_{E} \eta \phi_{\sigma}\left(\left[u_{j}-\llbracket T_{\ell}\left(u^{(\varepsilon)}\right) \rrbracket_{h}\right](x, T)\right) d x+\int_{E} \eta \phi_{\sigma}(0) d x\right] \leq 0,
\end{aligned}
$$

where the first term is less or equal than 0 by the definitions of $\phi_{\sigma}$ and $T_{\sigma}$, and the second integral disappears since $\phi_{\sigma}(0)=0$. As for the other contribution in (4.57), we use the fundamental property $\partial_{t} \llbracket T_{\ell}\left(u^{(\varepsilon)}\right) \rrbracket_{h}=\frac{1}{h}\left(T_{\ell}\left(u^{(\varepsilon)}\right)-\llbracket T_{\ell}\left(u^{(\varepsilon)}\right) \rrbracket_{h}\right)$ from $[21$, Lemma 2.2, p. 417], pass to the limit $j \rightarrow \infty$, and decompose $E_{T}$ into sets where $u$ is larger than $\ell, u$ is between $\varepsilon$ and $\ell$, and $u$ is less or equal than $\varepsilon$. Then, due to the fact that $T_{\sigma}$ is nondecreasing, there holds $\left(s_{1}-s_{2}\right) T_{\sigma}\left(s_{1}-s_{2}\right) \geq 0$ for any $s_{1}, s_{2} \in \mathbf{R}$ such that the integrals on $E_{T} \cap\{u>\ell\}$ and $E_{T} \cap\{\varepsilon<u \leq \ell\}$ can be estimated by 0 . Hence, we conclude that

$$
\begin{aligned}
\lim _{j \rightarrow \infty} \mathbf{L}_{\mathbf{2}}^{(1)} & =-\frac{1}{h} \iint_{E_{T}} \eta\left[T_{\ell}\left(u^{(\varepsilon)}\right)-\llbracket T_{\ell}\left(u^{(\varepsilon)}\right) \rrbracket_{h}\right] T_{\sigma}\left(u-\llbracket T_{\ell}\left(u^{(\varepsilon)}\right) \rrbracket_{h}\right) d z \\
& \leq-\frac{1}{h} \iint_{E_{T} \cap\{u \leq \varepsilon\}} \eta\left[\varepsilon-\llbracket \varepsilon \rrbracket_{h}\right] T_{\sigma}\left(u-\llbracket \varepsilon \rrbracket_{h}\right) d z \\
& \leq \frac{\sigma \varepsilon}{h} \int_{E} \int_{0}^{T} e^{-\frac{t}{h}} d t d x \leq c \sigma \varepsilon
\end{aligned}
$$

with a constant $c=|E|$. Merging (4.54)-(4.59), we infer from (4.53) that

$$
\lim _{h \searrow 0} \limsup _{j \rightarrow \infty} \mathbf{J}_{1}^{(-)} \leq c \sigma(1+\varepsilon)
$$

with a constant $c \equiv c\left(n, m, p, q, C_{0}, C_{1},\left|E_{T}\right|, \mu\left(E_{T}\right),\|D \eta\|_{L^{\infty}\left(E, \mathbf{R}^{n}\right)}\right)$ for almost every $\sigma>0$ and any $0<\varepsilon<\sigma$. As a consequence, if we insert (4.52) and (4.60) into (4.45) 
and choose $\sigma \in(\varepsilon, 2 \varepsilon)$, we have proved that

$$
\limsup _{\ell \rightarrow \infty} \lim _{h \searrow 0} \limsup _{j \rightarrow \infty} \mathbf{J}_{j, \ell}^{(-)}(\varepsilon, \sigma, h) \leq c \varepsilon^{\vartheta} .
$$

We will now put together the above results and show that $D u_{j}^{\alpha+1}$ subconverges to $D u^{\alpha+1}$ almost everywhere on $E_{T}$, we will argue that (4.69) is valid.

4.4.4. The conclusion. For $\varepsilon>0$, we obtain by (4.36), (4.40), (4.42), (4.44) and (4.61) that for $\mathbf{h}_{j}^{(\varepsilon)}$ as in (4.35) and $\vartheta$ as in (4.43), there holds

$$
\limsup _{j \rightarrow \infty} \iint_{E_{T}}\left[\eta \mathbf{h}_{j}^{(\varepsilon)}\right]^{\vartheta} d z \leq c \varepsilon^{\vartheta}
$$

for any $\eta \in C_{0}^{\infty}(E,[0,1])$ with a constant $c$ only depending on $n, m, p, q, C_{0}, C_{1}$, $C_{2},\left|E_{T}\right|, \mu\left(E_{T}\right)$ and $\|D \eta\|_{L^{\infty}\left(E, \mathbf{R}^{n}\right)}$. At this point, we pick a null sequence $\left(\varepsilon_{j}\right)_{j \in \mathbf{N}}$. Then, the last inequality yields

$$
\limsup _{j \rightarrow \infty} \iint_{E_{T}}\left[\eta \mathbf{h}_{j}^{\left(\varepsilon_{j}\right)}\right]^{\vartheta}=0 .
$$

From this, it follows at least for a subsequence, which we do not relabel, that

$$
\lim _{j \rightarrow \infty} \mathbf{h}_{j}^{\left(\varepsilon_{j}\right)}=0
$$

on $\widetilde{E}_{T}$ for a subset $\widetilde{E}_{T} \subset E_{T}$ with $\left|\widetilde{E}_{T}\right|=\left|E_{T}\right|$. Now, if $p \geq 2$, then $\lambda=p$, and (4.62) leads to

$$
\lim _{j \rightarrow \infty}\left|\frac{1}{\alpha+1} D u_{j}^{\alpha+1}-u_{j}^{\alpha} D u^{\left(\varepsilon_{j}\right)}\right|=0
$$

almost everywhere on $E_{T}$, where we may recall that $D u^{\left(\varepsilon_{j}\right)}$ has to be understood as recommended in (4.34). Our next aim is to show that (4.63) is valid also in the case $p \in\left(\frac{2 n}{n+2}, 2\right)$, in which $\lambda=2$. Since $D u^{\alpha+1} \in L^{q}\left(E_{T}, \mathbf{R}^{n}\right)$ for $q$ as in the range from (4.19), we can presume that $D u^{\alpha+1}$ is finite on $\widetilde{E}_{T}$ after possibly reducing $\widetilde{E}_{T}$ by a set of measure 0 , and we may further modify the set such that $\mathbf{b}(\cdot, u)<\infty$ on $\widetilde{E}_{T}$. Besides, by the convergence in (4.31), we can assume without loss of generality that $u_{j}(z) \rightarrow u(z)$ as $j \rightarrow \infty$ and $u(z)<\infty$ for any $z \in \widetilde{E}_{T}$. Hence, for a fixed $z \in \widetilde{E}_{T}$, we find

$$
\begin{aligned}
\mathbf{d}_{j}^{(\mathbf{1})}(z) & :=\left|u_{j}^{\alpha}(z) D u^{\left(\varepsilon_{j}\right)}(z)\right| \\
& =\left|u_{j}^{\alpha}(z) \frac{1}{\alpha+1} \chi_{E_{T} \cap\left\{u>\varepsilon_{j}\right\}}(z) u^{-\alpha}(z) D u^{\alpha+1}(z)\right| \leq c_{z}^{(1)}<\infty
\end{aligned}
$$

and (analogously, in view of the continuity of $\mathbf{b}(z, \cdot)$ )

$$
\mathbf{d}_{j}^{(2)}(z):=\left|u_{j}^{\alpha}(z) \mathbf{b}\left(z, u_{j}\right)\right| \leq c_{z}^{(2)}<\infty
$$

for any $j \gg 1$ (for the sake of brevity, we will omit the restriction $j \gg 1$, just saying $j \in \mathbf{N}$ ). The constants $c_{z}^{(1)}, c_{z}^{(2)}>0$ are independent of $j$. In other words, $\left(\mathbf{d}_{j}^{(\mathbf{1})}(z)\right)_{j \in \mathbf{N}}$ and $\left(\mathbf{d}_{j}^{(\mathbf{2})}(z)\right)_{j \in \mathbf{N}}$ are (nonuniformly) bounded at every point $z \in \widetilde{E}_{T}$. Additionally, for some fixed $M_{z}>0$, we are able to ensure that there holds

$$
c_{z}^{(1)} \leq \mathbf{d}_{j}^{(1)}(z)+\frac{1}{j} M_{z}
$$

for any $j \in \mathbf{N}$. We define

$$
\mathbf{d}_{j}^{(3)}(z):=\left|\frac{1}{\alpha+1} D u_{j}^{\alpha+1}(z)\right|
$$


for short. Then, using (4.64)-(4.66), we can estimate the function $\mathbf{h}_{j}^{\left(\varepsilon_{j}\right)}$ at $z \in \widetilde{E}_{T}$ as follows:

$$
\begin{aligned}
\mathbf{h}_{j}^{\left(\varepsilon_{j}\right)}(z) & \geq \frac{\left|\mathbf{d}_{j}^{(\mathbf{3})}(z)-\mathbf{d}_{j}^{(\mathbf{1})}(z)\right|^{2}}{c\left[\left(\mathbf{d}_{j}^{(\mathbf{3})}(z)^{p}+\mathbf{d}_{j}^{(\mathbf{2})}(z)^{p}\right)+\left(\mathbf{d}_{j}^{(\mathbf{1})}(z)^{p}+\mathbf{d}_{j}^{(\mathbf{2})}(z)^{p}\right)\right]^{\frac{2-p}{p}}} \\
& \geq \frac{\mathbf{d}_{j}^{(\mathbf{3})}(z)^{2}-2 c_{z}^{(1)} \mathbf{d}_{j}^{(\mathbf{3})}(z)+\left[c_{z}^{(1)}-\frac{1}{j} M_{z}\right]^{2}}{c_{z}\left[\mathbf{d}_{j}^{(3)}(z)+1\right]^{2-p}},
\end{aligned}
$$

where $c_{z}>0$ is a constant independent of $j$. Now, suppose that $\left(\mathbf{d}_{j}^{(\mathbf{3})}(z)\right)_{j \in \mathbf{N}}$ is unbounded at some point $z \in \widetilde{E}_{T}$. Consequently, for a nonrelabeled subsequence, we infer $\mathbf{d}_{j}^{(\mathbf{3})}(z) \rightarrow \infty$ as $j \rightarrow \infty$, hence (taking into account that $p>\frac{2 n}{n+2}$ ), we derive from (4.67) that $\mathbf{h}_{j}^{\left(\varepsilon_{j}\right)}(z) \rightarrow \infty$ as $j \rightarrow \infty$, which is a contradiction to (4.62). Thus, $\left(\mathbf{d}_{j}^{(3)}(z)\right)_{j \in \mathbf{N}}$ is (nonuniformly) bounded at every point $z \in \widetilde{E}_{T}$, and, since all terms appearing in the denominator of $\mathbf{h}_{j}^{\left(\varepsilon_{j}\right)}$ are (nonuniformly) bounded, we conclude from (4.62) that the numerator of $\mathbf{h}_{j}^{\left(\varepsilon_{j}\right)}$ tends to 0 on $\widetilde{E}_{T}$ as $j \rightarrow \infty$, i.e. (4.63) holds true for any $p>\frac{2 n}{n+2}$. Moreover, let us argue for any $p>\frac{2 n}{n+2}$ that

$$
\lim _{j \rightarrow \infty}\left|u_{j}^{\alpha} D u^{\left(\varepsilon_{j}\right)}-\frac{1}{\alpha+1} D u^{\alpha+1}\right|=0
$$

almost everywhere on $E_{T}$. To that end, first, pick an arbitrary $z \in E_{T}$ such that $u(z)>0$. By (1.11) and (4.31), we find

$$
u_{j}^{\alpha} D u-\frac{1}{\alpha+1} D u^{\alpha+1}=\frac{1}{\alpha+1}\left[u_{j}^{\alpha} u^{-\alpha}-1\right] D u^{\alpha+1} \rightarrow 0
$$

almost everywhere on $E_{T} \cap\{u>0\}$ as $j \rightarrow \infty$. Since $D u^{\left(\varepsilon_{j}\right)}(z)=D u(z)$ for any sufficiently small $\varepsilon_{j}>0$, this implies (4.68). On the other hand, if $z \in E_{T}$ and $u(z)=0$, then $D u^{\alpha+1}(z)=0$ and $D u^{\left(\varepsilon_{j}\right)}(z)=0$ for all $\varepsilon_{j}>0$ by (4.34). Combining (4.63) and (4.68), we finally obtain

$$
\lim _{j \rightarrow \infty}\left|\frac{1}{\alpha+1} D u_{j}^{\alpha+1}-\frac{1}{\alpha+1} D u^{\alpha+1}\right|=0
$$

almost everywhere on $E_{T}$, i. e. we have proved that

$$
D u_{j}^{\alpha+1} \rightarrow D u^{\alpha+1} \text { a.e. on } E_{T} \text { as } j \rightarrow \infty
$$

converges for some subsequence of $\left(D u_{j}^{\alpha+1}\right)_{j \in \mathbf{N}}$, which we will omit in the following for the sake of brevity. We are now able to show that $u$ satisfies (1.10).

4.5. Passage to the limit in the equation. As $u_{j}$ is a weak solution of the Cauchy-Dirichlet problem (4.4) for any $j \in \mathbf{N}$, by (4.5), we know that

$$
\iint_{E_{T}}\left[-u_{j} \partial_{t} \varphi+\mathbf{A}\left(x, t, u_{j}, D u_{j}\right) \cdot D \varphi-\mu_{j} \varphi\right] d z=0
$$

for any $\varphi \in C^{1}\left(\overline{E_{T}}\right)$ vanishing on $[\partial E \times(0, T)] \cup[\bar{E} \times\{T\}]$. The terms involving $u_{j}$ and $\mu_{j}$ converge due to (4.31) and (4.2). To reason that also the diffusion term converges, we take advantage of (4.69). Indeed, for $z \in E_{T}$ with $D u_{j}^{\alpha+1}(z) \rightarrow D u^{\alpha+1}(z)$ and $u_{j}(z) \rightarrow u(z)>0$ as $j \rightarrow \infty$, we have

$$
\begin{aligned}
\lim _{j \rightarrow \infty} \mathbf{A}\left(x, t, u_{j}(z), D u_{j}(z)\right) & =\lim _{j \rightarrow \infty} \mathbf{A}\left(x, t, u_{j}(z), \frac{1}{\alpha+1} \chi_{\left\{u_{j}>0\right\}}(z) u_{j}^{-\alpha}(z) D u_{j}^{\alpha+1}(z)\right) \\
& =\mathbf{A}\left(x, t, u(z), \frac{1}{\alpha+1} \chi_{\{u>0\}}(z) u^{-\alpha}(z) D u^{\alpha+1}(z)\right),
\end{aligned}
$$


and for $z \in E_{T}$ with $D u_{j}^{\alpha+1}(z) \rightarrow D u^{\alpha+1}(z)$ and $u_{j}(z) \rightarrow 0$ as $j \rightarrow \infty$, we apply (1.4) to find

$$
\lim _{j \rightarrow \infty}\left|\mathbf{A}\left(x, t, u_{j}(z), D u_{j}(z)\right)\right| \leq c \lim _{j \rightarrow \infty} u_{j}^{\alpha}(z)\left|D u_{j}^{\alpha+1}(z)\right|^{p-1}=0
$$

with a constant $c \equiv c\left(m, p, C_{1}\right)$. Hence, $\mathbf{A}\left(\cdot, u_{j}, D u_{j}\right)$ converges to $\mathbf{A}(\cdot, u, D u)$ almost everywhere on $E_{T}$ as $j \rightarrow \infty$. Now, for any $\delta>0$, Egorov's theorem provides a subset $K_{\delta} \subset E_{T}$ with $\left|E_{T} \backslash K_{\delta}\right|<\delta$ such that $\mathbf{A}\left(\cdot, u_{j}, D u_{j}\right) \rightarrow \mathbf{A}(\cdot, u, D u)$ converges uniformly on $K_{\delta}$ as $j \rightarrow \infty$. Furthermore, the integral on $E_{T} \backslash K_{\delta}$ can be made small by an adequate choice of $\delta$ because of the uniform $L^{1}\left(E_{T}, \mathbf{R}^{n}\right)$-bound (4.20) for $\mathbf{A}\left(\cdot, u_{j}, D u_{j}\right)$. Altogether, we can conclude that

$$
\lim _{j \rightarrow \infty} \iint_{E_{T}} \mathbf{A}\left(x, t, u_{j}, D u_{j}\right) \cdot D \varphi d z=\iint_{E_{T}} \mathbf{A}(x, t, u, D u) \cdot D \varphi d z
$$

holds true for any $\varphi \in C^{1}\left(\overline{E_{T}}\right)$ vanishing on $[\partial E \times(0, T)] \cup[\bar{E} \times\{T\}]$. Consequently, $u$ is a very weak solution of the Cauchy-Dirichlet problem (1.2), meaning that Theorem 1.4 is proved.

\section{References}

[1] Aronson, D. G.: The porous medium equation. - In: Nonlinear diffusion problems (Montecatini Terme, 1985), Lecture Notes in Math. 1224, Springer, Berlin, 1986, 1-46.

[2] Boccardo, L., A. Dall'Aglio, T. Gallouët, and L. Orsina: Nonlinear parabolic equations with measure data. - J. Funct. Anal. 147, 1997, 237-258.

[3] Boccardo, L., and T. GallouËT: Non-linear elliptic and parabolic equations involving measure data. - J. Funct. Anal. 87, 1989, 149-169.

[4] Boccardo, L., and T. GallouËt: Nonlinear elliptic equations with right hand side measures. - Comm. Partial Differential Equations 17, 1992, 641-655.

[5] Bögelein, V., F. DuzaAr, and U. Gianazza: Porous medium type equations with measure data and potential estimates. - SIAM J. Math. Anal. 45, 2013, 3283-3330.

[6] Bögelein, V., F. DuzaAR, and U. Gianazza: Very weak solutions of singular porous medium equations with measure data. - Commun. Pure Appl. Anal. 14, 2015, 23-49.

[7] Bögelein, V., F. Duzaar, and P. Marcellini: Parabolic systems with $p, q$-growth: A variational approach. - Arch. Ration. Mech. Anal. 210, 2013, 219-267.

[8] Caffarelli, L. A., and A. Friedman: Regularity of the free boundary of a gas flow in an $n$-dimensional porous medium. - Indiana Univ. Math. J. 29, 1980, 361-391.

[9] DiBenedetto, E.: Degenerate parabolic equations. - Universitext, Springer, New York, 1993.

[10] DiBenedetto, E., U. Gianazza, and V. Vespri: Harnack's inequality for degenerate and singular parabolic equations. - Springer Monogr. Math., Springer, New York, 2012.

[11] Duzane, F., and G. Mingione: Gradient estimates via non-linear potentials. - Amer. J. Math. 133, 2011, 1093-1149.

[12] Ivanov, A. V.: Hölder estimates for a natural class of equations of the type of fast diffusion. - J. Math. Sci. (N. Y.) 89, 1998, 1607-1630.

[13] Ivanov, A. V.: Regularity for doubly nonlinear parabolic equations. - J. Math. Sci. (N.Y.) $83,1997,22-37$.

[14] Ivanov, A. V., and P.Z. Mkrtychyan: Existence of Hölder continuous generalized solutions of the first boundary value problem for quasilinear doubly degenerate parabolic equations. - J. Soviet Math. 62, 1992, 2725-2740. 
[15] Ivanov, A. V., P.Z. Mkrtychyan, and W. JÄGer: Existence and uniqueness of a regular solution of the Cauchy-Dirichlet problem for a class of doubly nonlinear parabolic equations. - J. Math. Sci. (N. Y.) 84, 1997, 845-855.

[16] Ivanov, A. V., P.Z. Mkrtychyan, and W. JäGer: Erratum to: Existence and uniqueness of a regular solution of the Cauchy-Dirichlet problem for a class of doubly nonlinear parabolic equations. - J. Math. Sci. (N. Y.) 184, 2012, 786-787.

[17] Kalashnikov, A. S.: Some problems of the qualitative theory of non-linear degenerate secondorder parabolic equations. - Russian Math. Surveys 42, 1987, 169-222.

[18] KilpeläInen, T., and J. MalÝ: Degenerate elliptic equations with measure data and nonlinear potentials. - Ann. Sc. Norm. Super. Pisa Cl. Sci. (4) 19, 1992, 591-613.

[19] Kilpeläinen, T., and J. MalÝ: The Wiener test and potential estimates for quasilinear elliptic equations. - Acta Math. 172, 1994, 137-161.

[20] Kinnunen, J., and P. Lindqvist: Definition and properties of supersolutions to the porous medium equation. - J. Reine Angew. Math. 618, 2008, 135-168.

[21] Kinnunen, J., and P. Lindqvist: Pointwise behaviour of semicontinuous supersolutions to a quasilinear parabolic equation. - Ann. Mat. Pura Appl. (4) 185, 2006, 411-435.

[22] Kinnunen, J., and T. KuUsi: Local behaviour of solutions to doubly nonlinear parabolic equations. - Math. Ann. 337, 2007, 705-728.

[23] KuUsi, T., and G. Mingione: Gradient regularity for nonlinear parabolic equations. - Ann. Sc. Norm. Super. Pisa Cl. Sci. (5) 12, 2013, 755-822.

[24] Ladyženskaja, O. A., V. A. Solonnikov, and N. N. Ural'ceva: Linear and quasilinear equations of parabolic type. - Transl. Math. Monogr. 23, 1967, Amer. Math. Soc., Providence, RI.

[25] Lions, J.-L.: Quelques méthodes de résolution des problemes aux limites non linéaires. Dunod, Paris (1969).

[26] Lukkari, T.: The fast diffusion equation with measure data. - Nonlinear Differ. Equ. Appl. 19, 2012, 329-343.

[27] Lukkari, T.: The porous medium equation with measure data. - J. Evol. Equ. 10, 2010, $711-729$.

[28] Manfredi, J. J., and V. Vespri: Large time behavior of solutions to a class of doubly nonlinear parabolic equations. - Electron. J. Diff. Equ. 1994, 1994, 1-17.

[29] Porzio, M. M., and V. Vespri: Hölder estimates for local solutions of some doubly nonlinear degenerate parabolic equations. - J. Differential Equations 103, 1993, 146-178.

[30] SAVARÉ, G., and V. VESPRI: The asymptotic profile of solutions of a class of doubly nonlinear equations. - Nonlinear Anal. 22, 1994, 1553-1565.

[31] Siljander, J.: Boundedness of the gradient for a doubly nonlinear parabolic equation. - J. Math. Anal. Appl. 371, 2010, 158-167.

[32] Simon, J.: Compact sets in the space $L^{p}(0, T ; B)$. - Ann. Mat. Pura Appl. 146, 1987, 65-96.

[33] Sturm, S.: Pointwise estimates for porous medium type equations with low order terms and measure data. - Electron. J. Diff. Equ. 2015, 2015, 1-25.

[34] Sturm, S.: Existence of weak solutions of doubly nonlinear parabolic equations. - Preprint, 2017.

[35] Tedeev, A., and V. Vespri: Optimal behavior of the support of the solutions to a class of degenerate parabolic systems. - Interfaces Free Bound. 17, 2015, 143-156.

[36] VÁzquez, J. L.: Smoothing and decay estimates for nonlinear diffusion equations. - Oxford Lecture Ser. Math. Appl., Oxford Univ. Press, Oxford, 2006.

[37] Vázquez, J. L.: The porous medium equation. - Oxford Math. Monogr., Oxford Univ. Press, Oxford, 2007. 
[38] VESPRI, V.: Harnack type inequalities for solutions of certain doubly nonlinear parabolic equations. - J. Math. Anal. Appl. 181, 1994, 104-131.

[39] VESPRI, V.: On the local behaviour of solutions of a certain class of doubly nonlinear parabolic equations. - Manuscripta Math. 75, 1992, 65-80.

Received 30 January 2017 • Accepted 3 March 2017 